\title{
Die elektrische Leitfähigkeit von Kupfer unter besonderer Berücksichtigung der Anisotropie des Gitterschwingungsspektrums*
}

\author{
Von Helmut Bross \\ Aus dem Institut für theoretische und angewandte Physik der Technischen Hochschule Stuttgart \\ und Max-Planck-Institut für Metallforschung, Stuttgart \\ (Z. Naturforschg. 14 a, 560-580 [1959]; eingegangen am 11. Dezember 1958)
}

\begin{abstract}
Aus den Kopplungskonstanten für die Wechselwirkung zwischen nähergelegenen Gitterionen wird das Schwingungsspektrum von Kupfer gittertheoretisch berechnet. Es zeigt sich, daß die Frequenz und die Polarisationsvektoren sehr stark von der Richtung der Ausbreitungsvektoren der Schallschwingungen abhängig sind. Die Übergangswahrscheinlichkeit $W\left(\mathfrak{k}, \mathfrak{k}^{\prime}\right)$ für die Streuung der Elektronen an den Gitterschwingungen ist daher nicht nur vom Betrag des Differenzvektors $\left(\mathfrak{k}-\mathfrak{k}^{\prime}\right)$ abhängig. Zur Lösung des Transportproblems, welche mit der Annahme einer Relaxationszeit nicht möglich ist, wird ein Variationsverfahren angewandt, das früher für anisotrop streuende Gitterfehler abgeleitet wurde.

Die spezifischen Widerstände bei $T=20,4^{\circ} \mathrm{K}, 77,4^{\circ} \mathrm{K}$ und $273,15^{\circ} \mathrm{K}$ stimmen ziemlich gut mit den gemessenen Werten überein, wenn man in das Bardeensche Matrixelement für die Wechselwirkung zwischen den Leitungselektronen und Gitterionen den von Kамве angegebenen Wert für die Energie des Grundzustands des $\mathrm{Cu}^{+}$-Ions einsetzt. Wegen der starken Anisotropie der Übergangswahrscheinlichkeit können bei tiefen Temperaturen ziemlich große Abweichungen von der Matrhiessenschen Regel auftreten. Die von Magnuson, Palmer und Koenler an einem strahlungsgeschädigten Kristall beim Wechsel zu einer höheren Erholungstemperatur festgestellte sprunghafte Zunahme des Restwiderstandes wird quantitativ als eine solche Abweichung erklärt. Die Änderung des elektrischen Widerstandes im Magnetfeld stimmt im Temperaturbereich $20^{\circ} \mathrm{K}-50{ }^{\circ} \mathrm{K}$ mit den experimentellen Ergebnissen überein.
\end{abstract}

\section{Einleitung und Problemstellung}

Seit den Arbeiten von Sommerfeld ${ }^{1}$, Houston ${ }^{2}$ und $\mathrm{BLOCH}^{3}$ hat man die Transportvorgänge in Metallen qualitativ verstanden. In jüngster Zeit hat sich die Notwendigkeit ergeben, sich mit dieser Frage von neuem zu beschäftigen, weil die Leitfähigkeitsgrößen, insbesondere die elektrische Leitfähigkeit, leicht meßbar sind und sich für die Untersuchung von Gitterfehlstellen in Metallen sehr gut eignen. Bei der Auswertung der Experimente zeigt es sich nun, daß die üblicherweise gemachte Annahme der Additivität des elektrischen Widerstandes aus einem temperaturabhängigen Teil, der durch die Streuung der Elektronen an den Gitterschwingungen erzeugt wird, und einen temperaturunabhängigen Teil, dem sog. Restwiderstand, der durch statische Gitterfehler hervorgerufen wird, nicht streng gültig ist, sondern daß Abweichungen von dieser sog. Matthiessenschen Regel auftreten können, die mit der Blochschen Theorie nicht verstanden werden können.

* Dissertation, Technische Hochschule Stuttgart 1958.

1 A. Sommerfeld, Naturwiss. 15, 825 [1927]; Z. Phys. 47, 1 [1928].

2 W. V. Houston, Z. Phys. 48, 449 [1928]; Phys. Rev. 34, 279 [1929].

3 F. B Восн, Z. Phys. 52, 555 [1928]; 59, 208 [1930].

4 W. Kohn u. J. M. Luttinger, Phys. Rev. 108, 590 [1957].
Die Anwendung der galvanomagnetischen Effekte und der Thermokraft bei dem Studium der Gitterfehlstellen in Metallen ist heute noch wenig aufschlußreich, weil man die entsprechenden Phänomene in idealen Kristallen sehr unbefriedigend versteht. Es besteht daher der dringende Wunsch, zunächst die Transportvorgänge im ungestörten Kristall zu untersuchen. Die von B Boch durchgeführten, quantenmechanischen Berechnungen der Leitfähigkeitsvorgänge lassen sich in folgender Weise erweitern, wenn man im Rahmen einer Einelektronentheorie bleibt:

1. Strenge quantenmechanische Behandlung des Transportproblems, d. h. eine Verallgemeinerung des von Kohn und Lutringer ${ }^{4}$ für statische Gitterfehler abgeleiteten Verfahrens für die Streuung der Elektronen an Gitterschwingungen.

2. Berücksichtigung des Nichtgleichgewichts der Gitterschwingungen - ein Effekt, der nur bei tiefer Temperatur zu berücksichtigen ist ${ }^{5-8}$.

5 R. Peierls, Ann. Phys., Lpz. 4, 121 [1930] ; 5, 244 [1930]; 12, 154 [1932].

6 P. G. Klemens, Proc. Phys. Soc., Lond. A 64, 1030 [1951].

7 E. H. Sondheimer, Canad. J. Phys. 34, 1246 [1956].

8 I. I. Hanna u. E. H. Sondheimer, Proc. Roy. Soc., Lond. A 239, 247 [1957]. 
3. Die Leitungselektronen werden nicht als quasifrei behandelt. Zwischen der Einelektronenenergie $\varepsilon(\mathfrak{f})$ und dem Ausbreitungsvektor $\mathfrak{f}$ besteht also nicht der Zusammenhang *

$$
\varepsilon(\mathfrak{f})=\frac{\hbar^{2}}{2 m^{*}} \mathfrak{f}^{2},
$$

$2 \pi \hbar=$ Plancksche Konstante, $m^{*}$ effektive Masse der Leitungselektronen.

4. Das Schwingungsspektrum wird nicht mit Hilfe der Debyeschen Theorie, sondern mit der Gitterdynamik berechnet ${ }^{9}$.

In der vorliegenden Arbeit beschäftigen wir uns nur mit dem vierten der obengenannten Punkte, d. h. wir suchen die Frage zu beantworten, wie sich der Leitungsmechanismus ändert, wenn man die Streuung am „richtigen“ Gitterspektrum berücksichtigt. Es wird sich zeigen, daß dies im Temperaturbereich $15^{\circ} \mathrm{K}<T<50^{\circ} \mathrm{K}$ die wichtigste Erweiterung ist. Bei höheren Temperaturen scheinen die nichtsphärischen Energieflächen, insbesondere für die Änderung des elektrischen Widerstandes im Magnetfeld, wichtig zu werden.

Zunächst können wir zeigen, daß die Gitterschwingungen, selbst wenn die Frequenz $\omega(\mathfrak{q})$ nur vom Betrag des Ausbreitungsvektors $\mathfrak{q}$ abhängig ist (Debyesche Theorie), die Elektronen in Metallen nie völlig isotrop streuen. Durch Absorption eines Phonons mit Ausbreitungsvektor $\mathfrak{q}$ wird ein Elektron nur dann vom Zustand $\mathfrak{f}$ in den Zustand $\mathfrak{f}^{\prime}$ gestreut, wenn folgende Auswahlregel erfüllt ist:

$$
\mathfrak{l}^{\prime}-\mathfrak{l}=\mathfrak{q}+\AA_{n},
$$

wobei $\mathfrak{\Re}_{n}$ ein Gittervektor im reziproken Gitter ist. Durch diese Forderung wird erreicht, daß q ein reduzierter Ausbreitungsvektor bleibt, auch wenn die Differenz $\mathfrak{f}^{\prime}-\mathfrak{f}$ nicht mehr innerhalb der ersten Brillouin-Zone liegt. Für diese Prozesse, bei denen $\Re_{n} \neq 0$ ist, führt Peierls ${ }^{5}$ den Begriff Umklappprozesse ein. Mit Hilfe des reziproken Gitters kann man leicht zeigen, daß die Übergangswahrscheinlichkeit $W\left(\mathfrak{f}, \mathfrak{f}^{\prime}\right)$ bei Umklappprozessen nicht mehr vom Winkel zwischen $\mathfrak{f}$ und $\mathfrak{f}^{\prime}$, sondern auch von der Lage des Ausgangsvektors $\mathfrak{f}$ abhängig ist. Wie dies zustande kommt, sei am Beispiel eines kubisch-

\footnotetext{
* Der Einfluß der Abweichungen der Flächen konstanter Elektronenenergie von der Kugelform auf die Leitfähig. keitsvorgänge wurde schon von verschiedenen Autoren diskutiert :

R. Peierls, Z. Phys. 53, 255 [1929] ; Ann. Phys., Lpz. 10, 97 [1931]. - L. Davis, Phys. Rev. 56, 93 [1939]. - R. Olson u. S. Rodrigues, Phys. Rev. 108, 1212 [1957]. -
}

flächenzentrierten Kristalls erläutert, wenn die beiden Vektoren $\mathfrak{f}$ und $\mathfrak{f}^{\prime}$ in der 100-Ebene liegen (siehe Abb. 1, S. 562).

Endet die Differenz der beiden Vektoren $\mathfrak{f}$ und $\mathfrak{f}^{\prime}$ innerhalb der ersten Brillouin-Zone, dann sind $\mathfrak{f}^{\prime}-\mathfrak{f}$ und $\mathfrak{q}$ miteinander identisch. Die Frequenz der Schallwellen ist von

$$
|\mathfrak{q}|=\left|\mathfrak{f}^{\prime}-\mathfrak{f}\right|=\sqrt{k^{2}+k^{\prime 2}+2 k k^{\prime} \cos \Theta}
$$

abhängig; außerdem liefern nur die longitudinalen Wellen einen Beitrag zum elektrischen Widerstand. Ganz anders dagegen, wenn die Differenz $\mathfrak{f}^{\prime}-\mathfrak{f}$ außerhalb der ersten Brillours-Zone liegt! Zunächst ist die Frequenz der Schallwellen, selbst wenn der Winkel zwischen $\mathfrak{f}^{\prime}$ und $\mathfrak{f}^{\prime}$ konstant bleibt, auch noch von der Lage der beiden Vektoren abhängig; durch Umklappprozesse werden also die Leitungselektronen anisotrop gestreut. Außerdem können, weil $\mathfrak{f}^{\prime}-\mathfrak{f}$ nicht mehr parallel zu $\mathfrak{q}$ ist, die Elektronen auch von Transversalwellen gestreut werden. Da im allgemeinen die Transversalwellen eine kleinere Ausbreitungsgeschwindigkeit als die Longitudinalwellen besitzen, sind diese bei tiefen Temperaturen noch angeregt, während die Longitudinalwellen mit gleichem Ausbreitungsvektor schon lange eingefroren sind. Der zuletzt beschriebene Einfluß der Umklappprozesse auf die Temperaturabhängigkeit des elektrischen Widerstandes wurde von $\mathrm{Z}_{\text {IMAN }}{ }^{10} \mathrm{im}$ Fall des $\mathrm{Na}$ untersucht. Um die übliche Transporttheorie mit einer winkelunabhängigen Relaxationszeit anwenden zu können, beseitigte er die oben beschriebene Anisotropie dadurch, daß er für einen bestimmten Winkel $\Theta$ eine Mittelung über alle möglichen Richtungen im f-Raum durchführte. Mit seiner Methode ist dadurch keine Änderung der Leitfähigkeit im Magnetfeld $\mathrm{zu}$ erwarten. Auch BAILYN ${ }^{9}$, der sich etwas später mit demselben Problem befaßte, führte eine ähnliche Mittelbildung durch. Im Gegensatz zu Ziman untersuchte er jedoch nicht ein isotropes Medium, sondern einen kubischen Einkristall. In der Näherung langer Wellen läßt sich hier das Schwingungsspektrum als Funktion der elastischen Konstanten $c_{11}, c_{12}$ und $c_{44}$ berechnen. Nur für besondere Ausbreitungsrichtungen erhält man in diesem

Bis heute gibt es jedoch keine befriedigende Behandlung des Transportproblems bei nichtsphärischer Energiefläche.

9 M. Bailyn, Thesis Harvard University Cambridge 1956. M. Bailyn u. H. Brooks, Amer. Phys. Soc., Ser. II, 1, 300 [1956].

10 J. M. Ziman, Proc. Roy. Soc., Lond. A 226, 436 [1954]. 


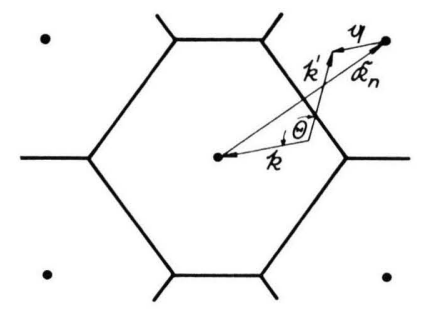

Abb. 1. Lage der Vektoren $\mathfrak{k}^{\prime}, \mathfrak{k}^{\prime}, \mathfrak{q}$ und $\Re_{n}$ bei einem Umklappprozeß.
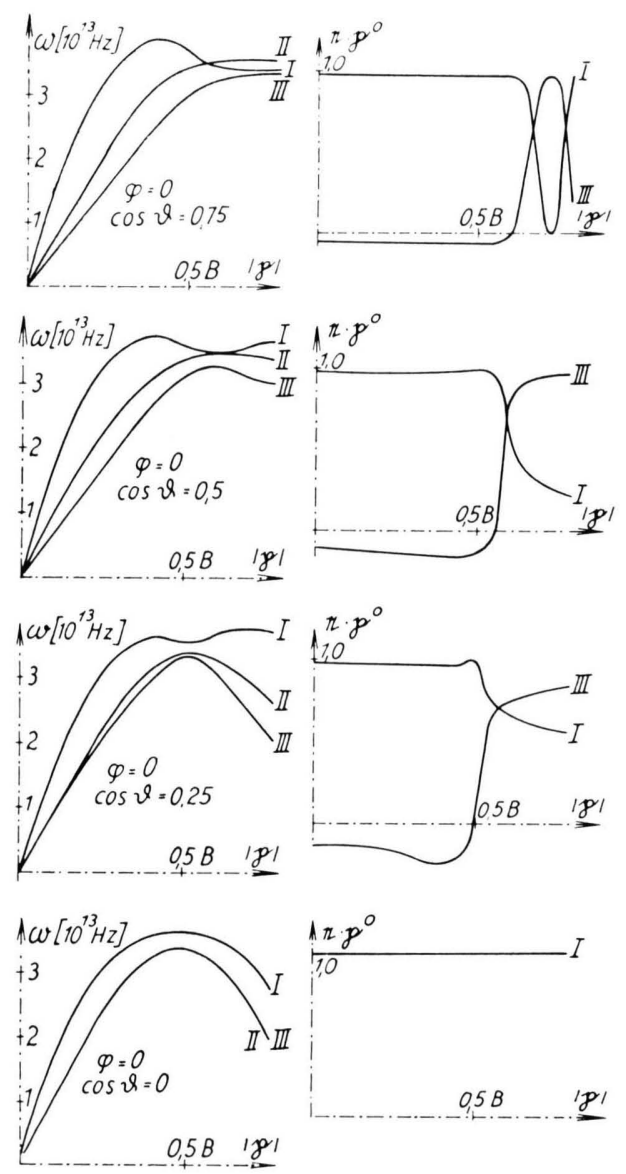

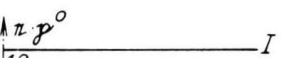$$
1 \pi \cdot 8^{\circ}
$$

1,0

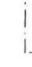
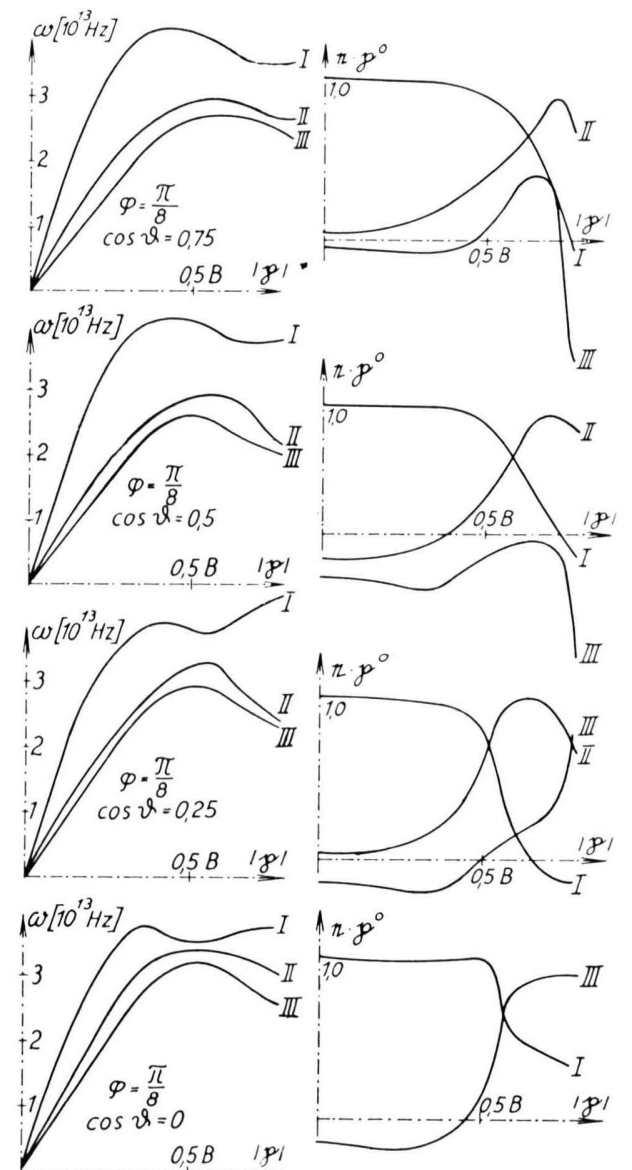

Abb. 2. Gitterschwingungsspektrum bei Kupfer.

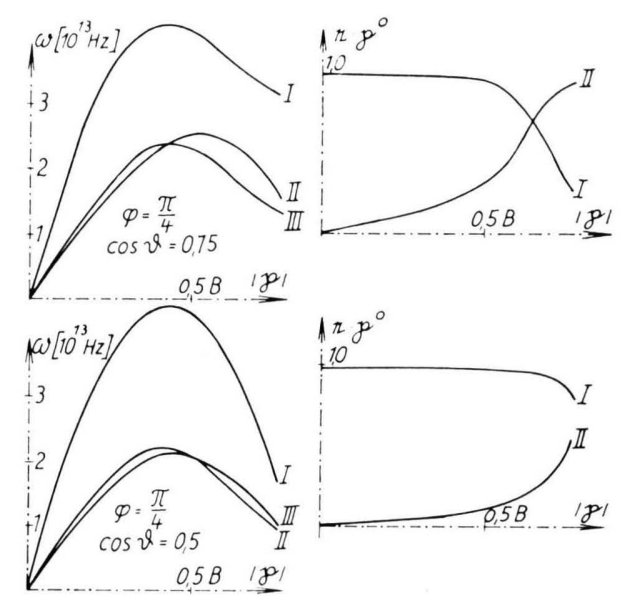

7
0
0
0
0
0
0
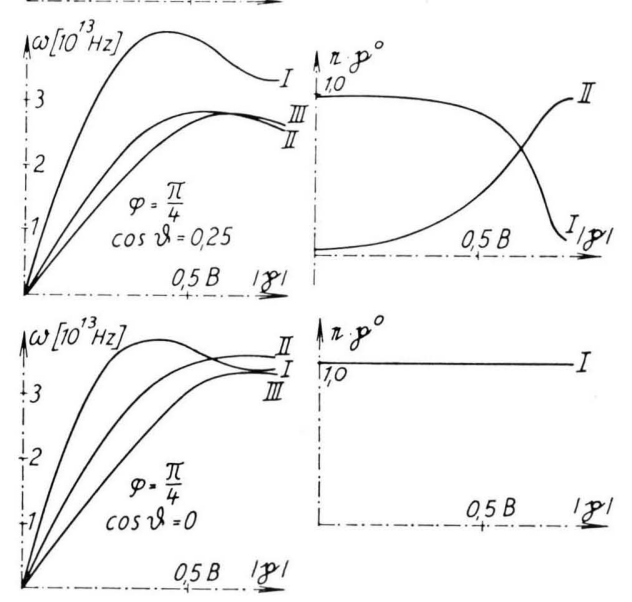
Modell reine longitudinale und reine transversale Wellen. In allen anderen Fällen bilden die aufeinander senkrecht stehenden Polarisationsvektoren einen beliebigen Winkel mit der Ausbreitungsrichtung. Da mit der Elastizitätstheorie nur das Frequenzspektrum langer Wellen richtig berechnet werden kann, führt er für die kürzeren Wellen einen empirischen Dispersionsfaktor ein, um dem wahren Spektrum im Kristall näher zu kommen. Wie bei ZIMAN wird die Richtungsanisotropie des Spektrums durch Mittelung eliminiert. Es ist daher möglich, die Transportgleichung mit Hilfe einer Relaxationszeit zu lösen. Obwohl er wegen dieser Vereinfachung die Änderung des elektrischen Widerstandes im Magnetfeld nicht berechnen konnte, waren seine Ergebnisse schon für den elektrischen Widerstand äußerst bemerkenswert: Oberhalb von etwa $1^{\circ} \mathrm{K}$ rührt der Hauptbeitrag zum temperaturabhängigen Widerstand bei den Alkalimetallen von den Umklappprozessen her. Außerdem erfolgt die Streuung der Elektronen bei tiefen Temperaturen nur durch die quasitransversalen Wellen, weil die quasilongitudinalen Wellen nicht mehr angeregt sind.

Die Arbeiten von Ziman und BaILyn haben also gezeigt, daß bei den Alkalimetallen die Anisotropie des Schwingungsspektrums einen großen Einfluß auf den elektrischen Widerstand hat. Es lag daher die Vermutung nahe, daß diese anisotropen Effekte auch für die Änderung des elektrischen Widerstandes im Magnetfeld und für die Abweichungen von der Matthiessenschen Regel verantwortlich sind. Wir haben diese Frage bei Kupfer näher untersucht, bei dem sich das Schwingungsspektrum mit Hilfe der Gitterdynamik exakt berechnen läßt, weil die zwischen den einzelnen Atomen wirkenden Kräfte aus den Messungen von $\mathrm{J}_{\mathrm{ACOBSEN}}{ }^{11}$ aus der diffusen Streuung von Röntgen-Strahlen bekannt sind. Unsere Rechnungen gehen darin über ZIMAN und BaILyN hinaus, daß über die Anisotropie der Übergangswahrscheinlichkeit nicht gemittelt wird. Dies ist unbedingt nötig, wenn man die typisch anisotropen Effekte, wie die Änderung des elektrischen Widerstandes im Magnetfeld und die Abweichungen von der Matthiessenschen Regel theoretisch erklären will, was mit den von den obigen Autoren angegebenen Verfahren nicht möglich ist. Das Transportproblem läßt sich dann nicht mehr mit dem üblichen, einfachen Ansatz für die Verteilungsfunktion $f(\mathfrak{f})$ der Elektronen lösen, bei dem man eine lineare $\mathrm{Ab}$ hängigkeit von den Komponenten des Ausbreitungs- vektors annimmt. Es müssen vielmehr noch höhere Potenzen der Komponenten des Ausbreitungsvektors berücksichtigt werden, was mit Hilfe eines Variationsverfahrens durchgeführt werden kann, das wir früher für anisotrop streuende, statische Gitterfehler abgeleitet haben ${ }^{12}$. Es wird sich zeigen, daß man auf diese Weise die Änderung des elektrischen Widerstandes im Magnetfeld, sowie Abweichungen von der Matthiessenschen Regel bei Kupfer im Temperaturbereich $15^{\circ} \mathrm{K}<T<50{ }^{\circ} \mathrm{K}$ verstehen kann. Bei hohen Temperaturen kann man dagegen die starken galvanomagnetischen Effekte nicht durch das anisotrope Gitterspektrum erklären, sondern es sind dafür sehr wahrscheinlich die nichtsphärischen Energieflächen verantwortlich.

\section{Formale Transporttheorie}

\section{a) Lösung des Transportproblems ohne Magnetfeld}

Wenn wir den Einfluß der Anisotropie des Gitterschwingungsspektrums auf die elektrische Leitfähigkeit untersuchen wollen, können wir die dabei auftretende Boltzmannsche Transportgleichung nicht mit der Annahme einer skalaren Relaxationszeit für die Elektron-Phonon-Streuung lösen, weil die Übergangswahrscheinlichkeit $W\left(\mathfrak{f}, \mathfrak{f}^{\prime}\right)$ für die Streuung der Elektronen nicht nur vom Ausdruck $\left|\mathfrak{f}-\mathfrak{f}^{\prime}\right|$ abhängig ist. Zur Lösung des Transportproblems für anisotrop streuende Medien wurde von Bross und SEEGER ${ }^{12}$ ein Variationsverfahren angegeben, das wir im folgenden auch benützen wollen. Hierbei treten des öfteren Integrale der Form

$$
\int \frac{\partial f_{0}}{\partial \varepsilon} g(\varepsilon)\left(\frac{\varepsilon-\zeta}{\zeta}\right)^{r} \mathrm{~d} \varepsilon
$$

mit $r<p$ auf. Die Untersuchungen von Kohler ${ }^{13}$, Jones ${ }^{14}$ und BAILyN ${ }^{9}$ haben gezeigt, daß der Fehler unter einem Prozent bleibt, wenn man bei der Berechnung des elektrischen Widerstandes keine Abhängigkeit der Verteilungsfunktion von der Energie annimmt, also $p=0$ setzt. Da in unserem Problem nur die Winkelabhängigkeit eine andere ist, beschränken wir uns später auch auf $p=0$.

11 E. H. Jacobsen, Phys. Rev. 97, 654 [1955].

12 H. Bross u. A. SeEger, J. Phys. Chem. Solids 4, 161 [1958].

13 M. Kohler, Z. Phys. 125, 679 [1948].

14 H. Jones, Handbuch der Physik (Flügge) XIX, SpringerVerlag, Berlin-Göttingen-Heidelberg 1956. 
b) Ableitung eines Variations-Iterationsverfahrens zur Lösung des Transportproblems mit Magnetfeld

Wenn außer dem elektrischen Feld auch noch ein Magnetfeld $\mathfrak{S}$ vorhanden ist, dessen Stärke jedoch nicht so groß sein soll, daß die Energieeigenwerte im Kristall verändert werden, ist die Verteilungsfunktion der Elektronen durch folgende Integrodifferentialgleichung bestimmt

$$
\mathfrak{L}\{\mathfrak{X}\}+\mathfrak{L}_{\mathfrak{D}}\{\mathfrak{X}\}=\frac{1}{\hbar} \frac{\partial f_{0}}{\partial \varepsilon} \operatorname{grad}_{\mathfrak{t}} \varepsilon,
$$

wobei $\Omega_{\mathfrak{H}}$ folgender Differentialoperator ist

$$
\mathfrak{L}_{\mathfrak{S}}\{\vec{\psi}(\mathfrak{f})\}=-\frac{e}{c \hbar^{2}} \frac{\partial f_{0}}{\partial \varepsilon}\left(\left[\operatorname{grad}_{\mathfrak{t}} \varepsilon \times \mathfrak{S}_{\mathfrak{L}}\right] \nabla_{\mathfrak{f}}\right) \vec{\psi}(\mathfrak{f}) .
$$

Im Gegensatz zum Operator $\mathfrak{Q}$ ist der Operator $\mathfrak{L}_{\mathfrak{H}}$ ein antisymmetrischer Operator im folgenden Sinne: Für zwei Funktionen $\psi_{1}(\mathfrak{f})$ und $\psi_{2}(\mathfrak{f})$ ist

$$
\frac{2}{(2 \pi)^{3}} \int \psi_{1} \Omega_{\mathfrak{H}}\left\{\psi_{2}\right\} \mathrm{d} \tau_{\mathfrak{f}}=-\frac{2}{(2 \pi)^{3}} \int \psi_{2} \Omega_{\mathfrak{H}}\left\{\psi_{1}\right\} \mathrm{d} \tau_{\mathfrak{f}} .
$$

Zum Beweis dieser Eigenschaft ${ }^{15,16}$ ist nur notwendig, daß die beiden Funktionen $\psi_{1}$ und $\psi_{2}$ im Unendlichen hinreichend verschwinden, was bei den im Zusammenhang mit der Leitfähigkeit auftretenden Funktionen $\mathfrak{X}(\mathfrak{f})$ immer der Fall ist.

Wegen der Asymmetrie des Operators $\mathfrak{L}_{\mathfrak{H}}$ kann zur Lösung der obigen Integrodifferentialgleichung nicht das früher beschriebene Variationsverfahren angewandt werden. Wenn die magnetische Feldstärke nicht allzu groß ist, läßt sich jedoch die obige Transportgleichung mit Hilfe eines Näherungsverfahrens lösen. In diesem Fall wird die Änderung der Elektronenverteilung vorwiegend durch die thermische Bewegung der Ionen und durch statische Gitterfehler erzeugt. Wir können daher den Operator $\mathfrak{Z}_{\mathfrak{5}}$ als Störung des Operators $\mathbb{R}$ auffassen:

$$
\Omega_{\mathfrak{5}}=\eta I_{\mathfrak{5}},
$$

wobei $\mathfrak{l}_{\mathfrak{5}}$ in derselben Größenordnung wie $\mathfrak{Z}$ ist.

Wir entwickeln ferner die Lösungen nach steigenden Potenzen des Störparameters

$$
\mathfrak{X}(\mathfrak{f})=\sum_{t=0}^{\infty} \mathfrak{X}^{(t)}(\mathfrak{f}),
$$

so $\operatorname{da} ß \mathfrak{X}^{(t+1)} \approx \eta \mathfrak{X}^{(t)}$ ist.

$$
\text { mit } \quad \mathfrak{S}^{(t)} \underset{r}{n}=\frac{2}{(2 \pi)^{3}} \frac{e}{c \hbar^{2}} \int \frac{\partial f_{0}}{\partial \varepsilon}\left\{\left(\left[\operatorname{grad} \varepsilon \times \mathfrak{S}_{\tau}\right]\right.\right.
$$

15 J. Meixner, Ann. Phys., Lpz. 40, 165 [1941].
Gehen wir mit diesen Ansätzen in die Integrodifferentialgleichung (4) ein, so ergibt sich folgendes Iterationssystem, weil der Störparameter $\eta$ beliebig ist,

$$
\begin{aligned}
t=0: \quad \mathfrak{L}\left\{\mathfrak{X}^{(0)}\right\} & =\frac{1}{\hbar} \frac{\partial f_{0}}{\partial \varepsilon} \operatorname{grad}_{\mathfrak{l}} \varepsilon, \\
t \geqq 1: \mathfrak{L}\left\{\mathfrak{X}^{(t)}\right\} & =-\mathfrak{L}_{\mathfrak{F}}\left\{\mathfrak{X}^{(t-1)}\right\} \\
& =\frac{e}{c \hbar^{2}} \frac{\partial f_{0}}{\partial \varepsilon}\left(\left[\operatorname{grad}_{\mathfrak{l}} \varepsilon \times \mathfrak{S}_{\mathfrak{g}}\right] \nabla_{\mathfrak{l}}\right) \mathfrak{X}^{(t-1)} .
\end{aligned}
$$

Die erste Gleichung entspricht der Integralgleichung für die Streuung der Elektronen ohne Magnetfeld; $\mathfrak{X}^{(0)}$ läßt sich daher mit Hilfe des Variationsverfahrens berechnen.

Aus der Integrodifferentialgleichung mit $t \neq 0$ kann man nun die richtige Verteilungsfunktion bei Anwesenheit eines Magnetfeldes iterativ berechnen, und zwar erhält man eine Entwicklung nach steigenden Potenzen von $\mathfrak{H}$. Im Sinne eines Iterationsverfahrens ist dabei immer die rechte Seite bekannt, während die Funktion $\mathfrak{X}^{(t)}$ mit Hilfe der Integralgleichung bestimmt werden soll. Da die gesuchte Funktion immer in Verknüpfung mit dem Operator $\mathfrak{Q}$ auftritt, können wir die iterativen Gleichungssysteme mit Hilfe des Variationsverfahrens berechnen. Wir führen dazu folgende Vergleichsfunktionen ein:

$\mathfrak{Y}^{(t ; p, q)}$

$$
=\sum_{r=0}^{p} \sum_{n=1}^{q} \sum_{m=-n}^{n} \mathfrak{Y}^{(t ; p, q)} \underset{r}{m}\left(\frac{\varepsilon-\zeta}{\zeta}\right)^{r} \Pi_{n}^{m}(\cos \vartheta) e^{i m \varphi},
$$

die noch den Nebenbedingungen

$$
\begin{aligned}
\frac{2}{(2 \pi)^{3}} \int \mathfrak{V}^{(t ; p, q)} & \mathbb{2}\left\{\mathfrak{Y}^{(t ; p, q)}\right\} \mathrm{d} \tau_{\mathfrak{t}} \\
= & -\frac{2}{(2 \pi)^{3}} \int \mathfrak{Y}^{(t ; p, q)} \Omega_{\mathfrak{S}}\left\{\mathfrak{X}^{(t-1)}\right\} \mathrm{d} \tau_{\mathfrak{t}}
\end{aligned}
$$

genügen müssen.

Nach Durchführung des Variationsverfahrens ergibt sich folgendes lineares Gleichungssystem für die unbekannten Entwicklungskoeffizienten

$$
\sum_{s=0}^{p} \sum_{l=1}^{\varphi} \sum_{o=-l}^{l} \mathfrak{Y}^{(t ; p, q)_{l}^{o}} \underset{s}{M_{n s}^{m o}}=\mathfrak{S}_{r s}^{(t)} \underset{r}{m},
$$




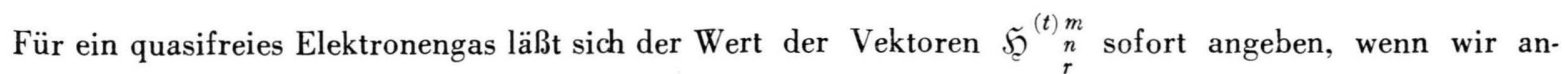
nehmen, daß wir die richtige Verteilungsfunktion $\mathfrak{X}^{(t-1)}(\mathfrak{f})$ in folgende unendliche Reihe entwickeln können

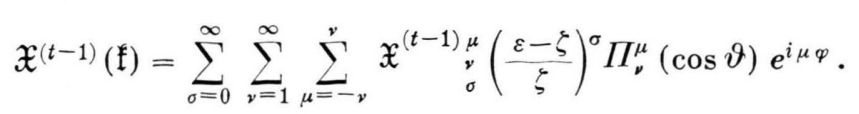

Bei Vernachlässigung höherer Potenzen im Entartungsparameter $(k T / \zeta)$ sind die allein für die elektrische Leitfähigkeit wichtigen Größen $\mathfrak{S}_{\mathfrak{Z}}{ }_{0}^{(t)} \underset{n}{n}$ gegeben durch

$$
\begin{aligned}
& \mathfrak{S}_{\substack{(t) \\
n \\
o}}^{m}=(-1)^{m} \frac{2}{(2 \pi)^{2}} \frac{e k \mathrm{~F}}{c \hbar^{2}}\left[\frac{1}{2}\left(i H_{x}+H_{y}\right) \sqrt{(n-m)(n+m+1)} \mathfrak{X}^{(t-1)-m-1} \underset{n}{n}\right. \\
& +\frac{1}{2}\left(\begin{array}{lll}
\left.i H_{x}-H_{y}\right) & \sqrt{(n-m+1)(n+m)} \mathfrak{X}^{(t-1)-m+1} \\
0 & +i m H_{z} & \mathfrak{X}^{(t-1)-m} \\
0
\end{array}\right] .
\end{aligned}
$$

In der Näherung quasifreier Elektronen ist der Vektor $\mathfrak{S C}_{r}^{(t) m} \underset{r}{m}$ nur von den Koeffizienten $\mathfrak{X}_{\substack{(t-1) m^{\prime} \\ n}}^{\text {mit }}$ demselben Index $n$ abhängig. Berechnen wir die Verteilungsfunktion bis zur $q$-ten Näherung in der Winkelabhängigkeit, so müssen nur die $\mathfrak{X}_{\substack{(t-1) m^{\prime} \\ n}}^{\substack{n \\ 0}}$ für $n \leq q$ bekannt sein. Bei hinreichend glatten Verteilungsfunktionen begeht man daher keinen großen Fehler, wenn man die Vergleichsfunktionen $\mathfrak{Y}^{(t-1 ; p, q)}$ für die Berechnung der $\mathfrak{S}_{c}^{(t) m}{ }_{0}^{m}$ benützt.

Für die numerische Berechnung der Änderung des elektrischen Widerstandes im Magnetfeld ist diese Näherung sehr gut geeignet: Dasselbe Gleichungssystem muß nämlich - nur mit abgeänderten rechten Seiten - mehrmals gelöst werden. Es liegt daher nahe, die zu den Koeffizientenmatrizen $\left(\begin{array}{c}m o \\ M n \\ r s\end{array}\right)$ gehörenden reziproken Matizizen $\begin{gathered}m o \\ i n \\ r s\end{gathered}$ einzuführen. Die Auflösung der linearen Gleichungssysteme für $t=0$ läßt sich formal ausdrücken durch

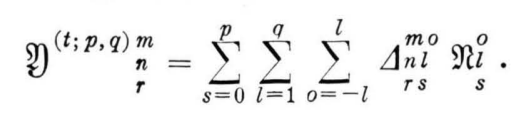

für $t \neq 0$ ist $\underset{s}{\mathfrak{R}_{l}^{o}}$ durch $\mathfrak{S}_{\substack{(t) \\ l}}^{o}$ zu ersetzen.

Wegen der obigen Eigenschaft ist das IterationsVariationsverfahren dem von Garcia-Molinar und Simons ${ }^{17}$ abgeleiteten Variationsverfahren überlegen, mit dem man zwar alle Leitfähigkeitsgrößen in einem Schritt berechnen kann, jedoch sehr viele Gleichungen bekommt, weil durch das äußere Feld die Symmetrie des Kristalls zerstört wird.

17 F. Garcia-Molinar u. S. Simons, Proc. Cambr. Phil. Soc. 53, 848 [1957]. c) Die Änderung des elektrischen Widerstandes im Magnetfeld bei einem Kristall mit tetragonaler Symmetrie

Bei einem Kristall von niederer Symmetrie führt schon das Variationsverfahren zur Berechnung des elektrischen Widerstandes zu einer Vielzahl von linearen Gleichungen, so daß eine Lösung des Transportproblems bei Vorhandensein eines äußeren Magnetfeldes praktisch unmöglich ist. Man muß sich daher, wenn man eine geschlossene Beziehung für die Leitfähigkeit im Magnetfeld erhalten will, auf einen Kristall mit möglichst hoher Symmetrie beschränken. Eine nähere Untersuchung zeigt, daß sich hierzu gut ein Kristall mit tetragonaler Symmetrie eignet, weil bei ihm nur jene Matrixelemente $\begin{gathered}m o \\ n l \\ r s\end{gathered}$ von Null verschieden sind, für die

$$
n+l=2 z \text { und } m+o=4 z^{\prime},
$$

wobei $z$ und $z^{\prime}$ beliebige ganze Zahlen sind. Außerdem ist

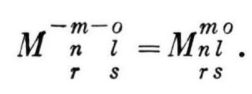

Da bei einem tetragonalen Kristall die Vektoren $\Re_{n}^{m}$ für gerades $n$ verschwinden, sind nur die Entwicklungskoeffizienten $\mathfrak{Y}_{r}^{m}{ }_{r}^{n+1}$ von Null verschieden; und zwar sind sie durch folgende linear unabhängige Gleichungssysteme bestimmt:

Gleichungssystem $\alpha: m=4 z, \quad o=4 z^{\prime}$, Gleichungssystem $\beta: m=1+4 z, \quad o=-2+4 z^{\prime}$, Gleichungssystem $\gamma: m=2+4 z, \quad o=-1+4 z^{\prime}$.

Bei tetragonaler Symmetrie zerfällt das Gleichungssystem (12) in drei voneinander unabhängige Glei- 
chungssysteme, die sich verhältnismäßig einfach lösen lassen. Andererseits ist diese Symmetrie noch so allgemein, daß man mit ihr neben allen Kristallen mit kubischer Symmetrie auch noch viele anisotrop statisch streuende Gitterfehler, z. B. Gitterfehler mit Rotationssymmetrie (u. a. Leerstellenpaare und Schraubenversetzungen), beschreiben kann.

Wie in einer früheren Arbeit ${ }^{18}$ gezeigt wurde, ge- nügt es selbst bei sehr anisotrop streuenden Gitterfehlern zur Beschreibung der Winkelabhängigkeit der Verteilungsfunktion bis zu Kugelflächenfunktionen dritter Ordnung einschließlich zu gehen. Eine längere Zwischenrechnung, bei der keine weiteren Überlegungen nötig sind, führt bei einem quasifreien Elektronengas auf folgende Beziehungen für die Elemente des Widerstandstensors ${ }^{19,} 20$

$$
\begin{aligned}
& \varrho_{x x}^{(0 ; 3)}=\varrho_{y y}^{(0 ; 3)}=-e^{-2} \delta^{-2} \frac{1}{\Delta_{11}^{1-1}}, \\
& \varrho_{z z}^{(0 ; 3)}=e^{-2} \delta^{-2} \frac{1}{\Delta_{11}^{90}} ; \\
& \varrho_{x y}^{(1 ; 3)}=-\varrho_{y x}^{(1 ; 3)}=e^{-2} \delta^{-2} \beta\left[1+\left(\frac{\Delta_{13}^{1-1}}{\Delta_{11}^{1-1}}\right)^{2}-3\left(\frac{\Delta_{13}^{13}}{\Delta_{11}^{1-1}}\right)^{2}\right] H_{z}, \\
& \varrho_{x z}^{(1 ; 3)}=-\varrho_{z x}^{(1 ; 3)}=-e^{-2} \delta^{-2} \beta\left[1+\sqrt{6} \frac{\Delta_{13}^{00}}{\Delta_{11}^{00}} \frac{\Delta_{13}^{1-1}}{\Delta_{11}^{1-1}}\right] H_{y}, \\
& \varrho_{y z}^{(1 ; 3)}=-\varrho_{z y}^{(1 ; 3)}=e^{-2} \delta^{-2} \beta\left[1+\sqrt{6} \frac{\Delta_{13}^{00}}{\Delta_{11}^{00}} \frac{\Delta_{13}^{1-1}}{\Delta_{11}^{1-1}}\right] H_{x} ; \\
& \varrho_{x x}^{(2 ; 3)}=e^{-2} \delta^{-2} \beta^{2}\left\{H_{x}^{2}\left(\Delta_{33}^{2-2}-\Delta_{33}^{22}\right)\left(\frac{\sqrt{10}}{2} \frac{\Delta_{13}^{1-1}}{\Delta_{11}^{1-1}}-\frac{\sqrt{6}}{2} \frac{\Delta_{13}^{13}}{\Delta_{11}^{1-1}}\right)^{2}\right. \\
& +H_{y}{ }^{2}\left[6\left(\Delta_{33}^{00}-\frac{\left(\Delta_{13}^{00}\right)^{2}}{\Delta_{11}^{00}}\right)\left(\frac{\Delta_{13}^{1-1}}{\Delta_{11}^{1-1}}\right)^{2}+\left(\Delta_{33}^{2-2}+\Delta_{33}^{22}\right)\left(\frac{\sqrt{10}}{2} \frac{\Delta_{13}^{1-1}}{\Delta_{11}^{1-1}}+\frac{\sqrt{6}}{2} \frac{\Delta_{13}^{13}}{\Delta_{11}^{1-1}}\right)^{2}\right] \\
& +H_{z}{ }^{2}\left[-\left(\Delta_{33}^{1-1}-\frac{\left(\Delta_{13}^{1-1}\right)^{2}}{\Delta_{11}^{1-1}}\right)\left(\frac{\Delta_{13}^{1-1}}{\Delta_{11}^{1-1}}\right)^{2}+6\left(\Delta_{33}^{13}-\frac{\Delta_{13}^{1-1} \cdot \Delta_{13}^{13}}{\Delta_{11}^{1-1}}\right) \frac{\Delta_{13}^{1-1}}{\Delta_{11}^{1-1}} \frac{\Delta_{13}^{13}}{\Delta_{11}^{1-1}}\right. \\
& \left.-9\left(\Delta_{33}^{3-3}-\frac{\left(\Delta_{13}^{13}\right)^{2}}{\Delta_{11}^{1-1}}\right)\left(\frac{\Delta_{13}^{13}}{\Delta_{11}^{1-1}}\right)^{2}\right\} \\
& \varrho_{z z}^{(2 ; 3)}=-e^{-2} \delta^{-2} \beta^{2} 6\left(\frac{\Delta_{13}^{00}}{\Delta_{11}^{00}}\right)^{2}\left(\Delta_{33}^{1-1}-\frac{\left(\Delta_{13}^{1-1}\right)^{2}}{\Delta_{11}^{1-1}}\right)\left(H_{x}{ }^{2}+H_{y}{ }^{2}\right), \\
& \varrho_{x y}^{(2 ; 3)}=\varrho_{y x}^{(2 ; 3)}=e^{-2} \delta^{-2} \beta^{2}\left\{\left(\Delta_{33}^{2-2}+\Delta_{33}^{22}\right)\left(\frac{\sqrt{10}}{2} \frac{\Delta_{13}^{1-1}}{\Delta_{11}^{1-1}}+\frac{\sqrt{6}}{2} \frac{\Delta_{13}^{13}}{\Delta_{11}^{1-1}}\right)^{2}\right. \\
& \left.-6\left(\Delta_{33}^{00}-\frac{\left(\Delta_{13}^{00}\right)^{2}}{\Delta_{11}^{00}}\right)\left(\frac{\Delta_{13}^{1-1}}{\Delta_{11}^{1-1}}\right)^{2}-\left(\Delta_{33}^{2-2}-\Delta_{33}^{22}\right)\left(\frac{\sqrt{10}}{2} \frac{\Delta_{13}^{1-1}}{\Delta_{11}^{1-1}}-\frac{l^{/ 6}}{2} \frac{\Delta_{13}^{13}}{\Delta_{11}^{1-1}}\right)^{2}\right\} H_{x} H_{y} . \\
& \varrho_{x z}^{(2 ; 3)}=\varrho_{z x}^{(2 ; 3)}=e^{-2} \delta^{-2} \beta^{2}\left\{\left(\Delta_{33}^{1-1}-\frac{\Delta_{13}^{1-1} \cdot \Delta_{31}^{1-1}}{\Delta_{11}^{1-1}}\right)^{2} \frac{\Delta_{13}^{1-1}}{\Delta_{11}^{1-1}}-3\left(\Delta_{33}^{13}-\frac{\Delta_{13}^{13} \cdot \Delta_{33}^{1-1}}{\Delta_{11}^{1-1}}\right) \frac{\Delta_{13}^{13}}{\Delta_{11}^{1-1}}\right\} \sqrt{6} \frac{\Delta_{13}^{00}}{\Delta_{11}^{00}} H_{x} H_{z}, \\
& \text { wobei } \quad \delta^{2}=\frac{1}{6 \pi^{4}} \frac{k_{\mathrm{F}}^{4}}{\hbar^{2}} \\
& \beta=\frac{1}{2 \pi^{2}} \frac{e k_{F}}{c \hbar^{2}} .
\end{aligned}
$$

Die Tensorelemente $Q_{y y}^{(2 ; 3)}$ etc. erhält man durch Vertauschen von $x$ und $y$.

Bei einem Kristall mit kubischer Symmetrie sind die Matrixelemente $\Delta_{n}^{m-o}$ nicht unabhängig voneinander, sondern durch folgende lineare Gleichun-

18 H. Bross u. A. Seeger, J. Phys. Chem. Solids 6, 324 [1958].

19 Durch Anwendung des Iterationsverfahrens erhält man den elektrischen Widerstand nach steigenden Potenzen des Magnetfeldes entwickelt

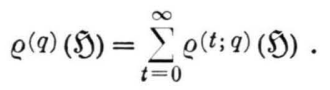

\footnotetext{
20 Im folgenden lassen wir das untere Indexpaar 00 weg.
}

gen miteinander gekoppelt ${ }^{21}$ :

$$
\begin{aligned}
\Delta_{11}^{00} & =-\Delta_{11}^{1-1}, \\
\sqrt{6} \Delta_{13}^{00} & =4 \Delta_{13}^{1-1}, \\
\sqrt{10} \Delta_{13}^{00} & =4 \Delta_{13}^{13},
\end{aligned}
$$

21 Die Richtigkeit der ersten drei Beziehungen ersieht man daraus, daß die durch ein elektrisches Feld erzeugte Änderung der Verteilungsfunktion sich in folgender Weise nach bikubisch-sphärischen Harmonischen entwickeln läßt.

$$
f(\mathfrak{E})-f_{0}(\mathfrak{E})=|\mathfrak{E}| \cdot \Sigma A_{1 n}^{(\varrho)}(k) \cdot B_{1 n}^{(\varrho)}\left(\vartheta_{0}, \varphi_{0} ; \vartheta, \varphi\right),
$$

wobei der E-Vektor durch die sphärischen Polarkoordinaten $|\mathfrak{E}|, \vartheta_{0}, \varphi_{0}$ festgelegt ist. 


$$
\begin{aligned}
\Delta_{33}^{00}+\Delta_{33}^{1-1}+\sqrt{\frac{10}{6}} \Delta_{33}^{13} & =0, \\
3 \Delta_{33}^{00}+8 \Delta_{33}^{1-1}+5\left(\Delta_{33}^{22}+\Delta_{33}^{2-2}\right) & =0, \\
2 \Delta_{33}^{00}-3 \Delta_{33}^{1-1}+5 \Delta_{33}^{3-3} & =0 .
\end{aligned}
$$

Zwischen der elektrischen Feldstärke \& einerseits und der elektrischen Stromdichte $\dot{i}$ und der magnetischen Feldstärke $\mathfrak{S}$ andererseits besteht in einem kubischen Kristall folgender phänomenologischer Zusammenhang ${ }^{22}$

$$
\mathfrak{F}=\varrho^{(0)}\left\{\mathrm{i}+a[\mathrm{j} \times \mathfrak{S}]+b \dot{\mathrm{j}} \mathfrak{S C}^{2}+c \mathfrak{S}(\mathrm{j} \mathfrak{S})+d \boldsymbol{P}, \mathrm{j}\right\},
$$

wenn man alle Glieder, die das Magnetfeld in einer höheren Potenz als der zweiten enthalten, vernachlässigt. $\boldsymbol{P}$ ist eine Diagonalmatrix mit den Elementen $H_{x}{ }^{2}, H_{y}{ }^{2}$ und $H_{z}{ }^{2}$. Alle Vektoren sind auf das Hauptachsensystem des kubischen Kristalls bezogen.

Bei einem quasifreien Elektronengas mit anisotropen Streumechanismen lassen sich die phänomenologisch eingeführten galvanomagnetischen Koeffizienten aus den Gln. (18) bis (22) berechnen.

$$
\begin{aligned}
\varrho^{(0)} & =e^{-2} \delta^{-2} \frac{1}{\Delta_{11}^{00}}, \\
a \varrho^{(0)} & =-e^{-2} \delta^{-2} \beta\left[1-\frac{3}{2}\left(\frac{\Delta_{13}^{00}}{\Delta_{11}^{00}}\right)^{2}\right], \\
b & =6 \beta^{2}\left(\frac{\Delta_{13}^{00}}{\Delta_{11}^{00}}\right)^{2}\left[\Delta_{11}^{1-1} \Delta_{33}^{1-1}-\Delta_{13}^{1-1} \Delta_{31}^{1-1}\right],(24 \mathrm{c}) \\
c={ }_{8}^{3} & \beta^{2}\left(\frac{\Delta_{13}^{00}}{\Delta_{11}^{00}}\right)^{2}\left[10 \Delta_{11}^{00}\left(\Delta_{33}^{2-2}+\Delta_{33}^{22}\right)\right. \\
d & \left.-6\left(\Delta_{11}^{00} \Delta_{33}^{00}-\Delta_{13}^{00} \Delta_{31}^{00}\right)\right], \\
& =-(b+c) .
\end{aligned}
$$

Unter den oben gemachten Annahmen verschwindet also die longitudinale Widerstandsänderung der Leitfähigkeit in Richtung der Hauptachsen des Kristalls.
Die Änderung des elektrischen Widerstandes im Magnetfeld läßt sich mit Hilfe der galvanomagnetischen Koeffizienten in folgender Form ausdrücken:

$$
\begin{aligned}
\Delta \varrho / \varrho^{(0)}= & b H^{2}+c\left(H_{1} \cos \alpha_{1}+H_{2} \cos \alpha_{2}+H_{3} \cos \alpha_{3}\right)^{2} \\
& +d\left(H_{1}{ }^{2} \cos ^{2} \alpha_{1}+H_{2}{ }^{2} \cos ^{2} \alpha_{2}+H_{3}^{2} \cos ^{2} \alpha_{3}\right),
\end{aligned}
$$

wenn die Stromdichte mit den Kristallhauptachsen den Winkel $\alpha_{1}, \alpha_{2}, \alpha_{3}$ bildet.

Ein polykristalliner Körper besteht aus einer Vielzahl von Einkristallen. Das magnetische Feld und der Stromvektor bilden daher alle möglichen Winkel mit den Hauptachsen der einzelnen Kristalle. Als Funktion der galvanomagnetischen Koeffizienten des Einkristalls bekommt man die longitudinale bzw. transversale Änderung des elektrischen Widerstandes im Magnetfeld, wenn man über alle diese Richtungen mittelt:

$$
\begin{aligned}
& A \|=\frac{\overline{\Delta \varrho \|}}{\varrho(0)}=\left(b+c+\frac{3}{5} d\right) H_{\| 1}^{2}, \\
& A_{\perp}=\frac{\overline{\Delta \varrho \perp}}{\varrho^{(0)}}=\left(b+\frac{1}{5} d\right) H_{\perp}^{2} .
\end{aligned}
$$

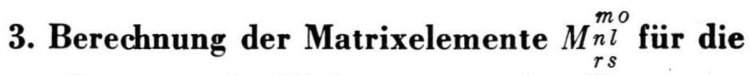 Streuung der Elektronen an den Gitter- schwingungen}

Unter der Annahme, daß sich Leitungselektronen durch Einelektronenfunktionen beschreiben lassen, hat Bloch die Wechselwirkung zwischen ihnen und den Gitterschwingungen mit Hilfe der Diracschen Störungstheorie berechnet. Die Wahrscheinlichkeit, $\mathrm{da} ß$ in der Zeiteinheit eines der beiden Elektronen mit Ausbreitungsvektor 1 durch Streuung an den Gitterschwingungen nach $\mathfrak{f}^{\prime}$ kommt, ist nach SommerFELD und BETHE gegeben durch ${ }^{23,} 24$

$$
W\left(\mathfrak{f}, \mathfrak{f}^{\prime}\right)=\frac{n^{2}}{\gamma_{0} V_{0}} \sum_{j} \frac{\left|\left(\mathfrak{e}_{\mathrm{q} j} \cdot \Im\left(\mathfrak{E}, \mathfrak{k}^{\prime}\right)\right)\right|^{2}}{2 \hbar \omega_{\mathrm{q} j}}\left\{\frac{\partial}{\partial t} \Omega\left(\varepsilon_{\mathfrak{t}^{\prime}}-\varepsilon \mathfrak{t}+\hbar \omega_{\mathrm{q}_{j} j}\right)\left(N_{\mathrm{q}}+1\right)+\frac{\partial}{\partial t} \Omega\left(\varepsilon \mathfrak{k}^{\prime}-\varepsilon \mathfrak{t}-\hbar \omega_{\mathrm{q} j}\right) N_{\mathrm{q}}\right\} .
$$

Mit dem ersten Glied werden Prozesse beschrieben, bei denen das Elektron den Zustand $\mathfrak{f}^{\prime}$ unter

22 Die Komponentendarstellung dieser Gleichung wurde von M. KoHler, Ann. Phys., Lpz. 20, 891 [1934] zuerst angegeben. Eine ähnliche Gleichung findet sich viel später bei F. SeItz, Phys. Rev. 79, 372 [1950].

23 A. Sommerfeld u. H. Bethe, Handbuch der Physik (GeigerSCHEEL) XXIV/2, Springer-Verlag, Berlin 1933.
Abgabe eines Schwingungsquants erreicht, es ist $\mathfrak{f}^{\prime}=\mathfrak{f}-\mathfrak{q}+\Re_{n}$. Beim zweiten Glied nimmt das Elek-

24 Da wir kein DeBYesches Schwingungsspektrum verwenden, müssen wir an Stelle der skalaren Größe $C$ die vektorielle Größe $\mathcal{J}\left(\mathfrak{f}, \mathfrak{k}^{\prime}\right)$ (vgl. l. c. ${ }^{23}$, Gl. 34.23 und 34.15 ) benützen, um die Wechselwirkung zwischen den Elektronen und Phononen zu beschreiben. Außerdem müssen wir noch über die drei Polarisationsrichtungen summieren. 
tron ein Schwingungsquant auf, es ist $\mathfrak{f}^{\prime}=\mathfrak{f}+\mathfrak{q}+\mathfrak{\Omega}_{n}$.

Dabei ist

$n$ Zahl der Leitungselektronen/Volumeinheit,

$\omega_{\mathrm{q} j}$ Kreisfrequenz der Gitterschwingung mit Ausbreitungsvektor $q$ und Polarisation $j$,

$e_{\mathrm{q} j}$ Einheitsvektor für die $j$-te Polarisationsrichtung,

$\gamma_{0}$ Dichte des Kristalls,

$N_{\mathrm{q}}$ Zahl der Gitterschwingungen mit Ausbreitungsvektor $\mathfrak{q}$.

$$
\frac{\partial \Omega(x)}{\partial t}=2 \frac{\sin (x / \hbar) t}{x / \hbar} .
$$

Es ist $\quad \int_{-\infty}^{\infty} f(x) \frac{\partial \Omega(x)}{\partial t} \mathrm{~d} x=2 \pi \hbar f(0)$.

Die in Gl. (27) vorkommende Vektorfunktion $\mathfrak{\Im}\left(\mathfrak{f}, \mathfrak{f}^{\prime}\right)$ ist definiert durch

$$
\Im\left(\mathfrak{f}, \mathfrak{f}^{\prime}\right)=\int_{\text {Kristall }} \psi_{\mathrm{t}^{\prime}}(\mathfrak{r}) \operatorname{grad} U(\mathfrak{r}) e^{i q \mathfrak{r}} \psi_{\mathfrak{t}}(\mathfrak{r}) \mathrm{d} \tau_{\mathfrak{r}},
$$

wenn man mit Sommerfeld-Bethe annimmt, daß die Metallionen durch die Gitterschwingungen deformiert werden und $U(\mathrm{r})$ das periodische Gitterpotential im ungestörten Kristall ist. Benützt man dagegen die von Nordherm ${ }^{25}$ eingeführte Näherung starrer Ionen, dann ist

$\mathfrak{\Im}\left(\mathfrak{f}, \mathfrak{f}^{\prime}\right)=\sum_{n} \int \psi_{\mathfrak{t}^{\prime}}^{*}(\mathfrak{r}) e^{i q \Re_{n}} \operatorname{grad} u\left(\mathfrak{r}-\Re_{n}\right) \psi_{\mathfrak{l}}(\mathfrak{r}) \mathrm{d} \tau_{\mathfrak{r}}$,

wobei die Summe über alle Ortsvektoren $\Re_{n}$ geht, welche die Gleichgewichtslagen der Ionen bestimmen; $u(\mathrm{r})$ ist das Potential eines Metallions. Sowohl Sommerfeld-Bethe als auch Nordheim haben bei ihren Untersuchungen nicht berücksichtigt, daß die Leitungselektronen das durch die Gitterschwingungen hervorgerufene Störpotential abschirmen können. Wie BARDEen ${ }^{26}$ später mit Hilfe einer selfconsistent-field-Methode gezeigt hat, kann man diese Abschirmung bei einem freien Elektronengas durch einen von $\mathfrak{f}$ und $\mathfrak{f}^{\prime}$ abhängigen Faktor $S\left(\mathfrak{f}, \mathfrak{f}^{\prime}\right)$ berücksichtigen, um den das „starre“ Ionenpotential $u(\mathrm{r})$ verringert wird. Für den gesuchten Vektor
$\mathfrak{\Im}\left(\mathfrak{f}, \mathfrak{f}^{\prime}\right)$ hat BARDEEN bei nahezu freien Elektronen in Hartreescher Näherung folgende Abhängigkeit abgeleitet:

$$
\begin{gathered}
\mathfrak{J}\left(\mathfrak{f}, \mathfrak{f}^{\prime}\right)=\frac{i}{S\left(\mathfrak{f}, \mathfrak{k}^{\prime}\right)} \frac{\mathfrak{k}^{-}-\mathfrak{k}^{\prime}}{n}\left(\frac{4 \pi e^{2} n}{\left|\mathfrak{k}-\mathfrak{k}^{\prime}\right|^{2}}+U_{0}{ }^{\prime}\left(r_{\mathrm{8}}\right)-E_{0}{ }^{\prime}\right) \\
\cdot \frac{3(\sin x-x \cdot \cos x)}{x^{3}}, \\
S\left(\mathfrak{f}, \mathfrak{f}^{\prime}\right)=1+\frac{8 \pi e^{2} n}{\left|\mathfrak{k}-\mathfrak{k}^{\prime}\right|^{2}} \frac{1}{w\left(\left|\mathfrak{k}-\mathfrak{k}^{\prime}\right|\right)}, \\
w(K)=\frac{4}{3} \frac{\hbar^{2} k_{F}^{2}}{2 m^{*}}\left\{\frac{1}{2}+\frac{4 k_{\mathrm{F}}^{2}-K^{2}}{8 k_{\mathrm{F}} K} \log \frac{2 k_{\mathrm{F}}+K}{2 k_{\mathrm{F}}-K}\right\}^{-1} .
\end{gathered}
$$

Hierbei ist

$k_{\mathrm{F}} \quad$ Ausbreitungsvektor der Elektronen an der FeRMIOberfläche,

$x=\left(\left|\mathfrak{k}-\mathfrak{k}^{\prime}\right|\right) r_{\mathrm{s}}$,

$r_{\mathrm{s}} \quad$ Radius der Wigner-Sertz-Kugel. Es ist $(4 \pi / 3) r_{\mathrm{s}}{ }^{3}=1 / n$.

$U_{0}^{\prime}(r)$ setzt sich additiv aus dem Ionenpotential $u(r)$ und dem Potential eines Leitungselektrons zusammen, welches das Gitterion abschirmt.

$U_{0}^{\prime}\left(r_{\mathrm{s}}\right)$ ist nahezu gleich Null.

$E_{0}^{\prime} \quad$ ist der Eigenwert folgender SchröDinger-Gleichung

$$
-\frac{\hbar^{2}}{2 m} \Delta \chi+U_{0}{ }^{\prime} \chi=E_{0}{ }^{\prime} \chi \text {. }
$$

Wenn $\chi$ innerhalb der Wigner-Sertz-Zelle nahezu konstant ist, dann ist $E_{0}{ }^{\prime}$ etwa um $1,2 e^{2} / r_{\mathrm{s}}$ größer als die Energie $E_{0}$ des Grundzustandes eines Elektrons, das sich im Felde des Metallions bewegt.

Für die weiteren Untersuchungen machen wir die Annahme, deren Berechtigung von verschiedenen Autoren ${ }^{5-8}$ eingehend untersucht worden ist, $\mathrm{da} ß$ auch unter Einfluß eines äußeren Feldes die Gitterschwingungen im thermischen Gleichgewicht bleiben, d. h. daß wir für $N(q)$ das Plancksche Gesetz anwenden können

$$
\begin{gathered}
N_{\mathfrak{q}}=1 /\left(\exp \left\{\hbar \omega_{\mathfrak{q} j} / k T\right\}-1\right), \\
N_{\mathfrak{q}}+1=1 /\left(1-\exp \left\{-\hbar \omega_{\mathfrak{q} j} / k T\right\}\right) .
\end{gathered}
$$

Die für die Leitfähigkeit wichtigen Matrixelemente $M_{n l}^{m o}$ sind wegen (27) bestimmt durch

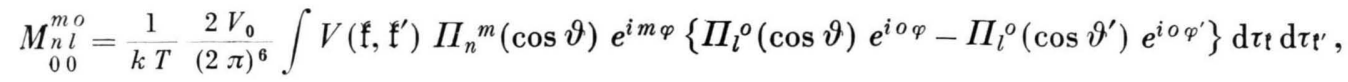

wobei

$$
\begin{aligned}
& V\left(\mathfrak{f}, \mathfrak{f}^{\prime}\right)=\frac{\pi \hbar}{\gamma_{0} V_{0}} n^{2} \sum_{j} \frac{1}{\exp \left\{\left(\varepsilon_{\mathfrak{f}}^{\prime}-\zeta\right) / k T\right\}+1} \frac{1}{1+\exp \left\{-\left(\varepsilon \varepsilon^{\prime}-\zeta\right) / k T\right\}} \frac{\mid\left(\left.\mathfrak{e}_{\mathrm{q} j} \cdot \mathcal{J}\left(\mathfrak{k}, \mathfrak{k}^{\prime}\right)\right|^{2}\right.}{\hbar \omega_{\mathrm{q} j}} \\
& { }^{25} \text { L. W. Nordheim, Ann. Phys., Lpz. 9, } 607 \text { [1931]. } \\
& 26 \text { J. BardeEn, Phys. Rev. 52, } 688 \text { [1937]. } \\
& \cdot\left\{\frac{\delta\left(\varepsilon_{\mathrm{t}}-\varepsilon_{\mathfrak{\ell}}^{\prime}-\hbar \omega_{\mathrm{a} j}\right)}{\exp \left\{\hbar \omega_{\mathrm{a} j} / k T\right\}-1}+\frac{\delta\left(\varepsilon_{\ell}-\varepsilon_{\ell}^{\prime}+\hbar \omega_{\mathrm{a} j}\right)}{1-\exp \left\{-\hbar \omega_{\mathrm{a} j} / k T\right\}}\right\}
\end{aligned}
$$


ist. Zunächst führen wir nur die Integration über $\varepsilon$ aus, wobei wir langsam veränderliche Größen wie $k(\varepsilon)$ und $\mathfrak{\Im}\left(\mathfrak{f}, \mathfrak{f}^{\prime}\right)$ als konstant betrachten und durch ihre Werte an der Stelle $\varepsilon=\zeta$ ersetzen können. Die schnell veränderlichen Terme formen wir mit Hilfe der Bedingungen, die von den $\delta$-Funktionen gestellt werden, noch etwas um. Für $\varepsilon \mathfrak{p}^{\prime}=\varepsilon \uparrow-\hbar \omega_{q_{j}}$ gilt folgende Identität

$$
\begin{aligned}
\int_{0}^{\infty} \frac{\mathrm{d} \varepsilon}{\left[\exp \left\{\left(\varepsilon \varepsilon^{\prime}-\zeta\right) / k T\right\}+1\right][1+\exp \{-(\varepsilon \mathrm{q}-\zeta) / k T\}]} \\
=\frac{1}{\exp \left\{-\hbar \omega_{\mathrm{a} j} / k T\right\}-1} \int_{0}^{\infty} \mathrm{d} \varepsilon\left\{\frac{1}{\exp \{(\varepsilon \mathrm{t}-\zeta) / k T\}+1}-\frac{1}{\exp \left\{\left(\varepsilon \mathrm{t}-\zeta-\hbar \omega_{\mathrm{q} j}\right) / k T\right\}+1}\right\} .
\end{aligned}
$$

Die auf der rechten Seite auftretenden Integrale lassen sich elementar ausführen ${ }^{27}$. Wenn $\zeta \gg k T$ ist, besitzt das Integral folgenden Wert

$$
\int_{0}^{\infty}\left[\exp \left\{\left(\varepsilon \varepsilon^{\prime}-\zeta\right) / k T\right\}+1\right]^{-1}[1+\exp \{-(\varepsilon-\zeta) / k T\}]^{-1} \mathrm{~d} \varepsilon=\hbar \omega_{\mathrm{q} j}\left[1-\exp \left\{-\hbar \omega_{\mathrm{q} j} / k T\right\}\right]^{-1} .
$$

Für $\varepsilon_{\mathfrak{t}^{\prime}}=\varepsilon \mathfrak{t}+\hbar \omega_{\mathfrak{q} j}$ brauchen wir nur $\omega_{\mathfrak{q}_{j}}$ durch $-\omega_{\mathfrak{q}_{j}} \mathrm{zu}$ ersetzen. Nach Integration über $\varepsilon$ erhalten wir demnach

$$
\begin{aligned}
& M_{n l}^{m o}=\frac{1}{(2 \pi)^{5}} \frac{n^{2}}{\gamma_{0}} \frac{\hbar}{k T} \sum_{j} \int\left|\left(e_{\mathrm{q} j} \cdot \mathfrak{J}\left(\mathfrak{f}, \mathfrak{f}^{\prime}\right)\right)\right|^{2} \frac{\delta\left(\varepsilon \mathfrak{p}-\varepsilon^{\prime}-\hbar \omega_{\mathrm{a} j}\right)+\delta\left(\varepsilon \mathfrak{q}-\varepsilon_{\mathfrak{\ell}}^{\prime}+\hbar \omega_{\mathrm{a} j}\right)}{\left(\exp \left\{\hbar \omega_{\mathrm{a} j} / k T\right\}-1\right)\left(1-\exp \left\{-\hbar \omega_{\mathrm{a} j} / k T\right\}\right)} \\
& \text { - } \Pi_{n}{ }^{m}(\cos \vartheta) e^{i m \varphi}\left\{\Pi_{l}{ }^{o}(\cos \vartheta) e^{i o \varphi}-\Pi_{l}^{o}\left(\cos \vartheta^{\prime}\right) e^{i o \varphi^{\prime}}\right\} k^{2} \frac{\partial k}{\partial \varepsilon} \mathrm{d} \omega_{l} \mathrm{~d} \tau_{\ell^{\prime}} .
\end{aligned}
$$

Alle von $\varepsilon$ abhängigen Größen sind an der Stelle $\varepsilon=\zeta$ zu nehmen. Bei der nun folgenden Integration über $\varepsilon^{\prime}$ liefern nur die Stellen $\varepsilon \mathfrak{f}^{\prime}=\zeta \pm \hbar \omega_{\mathrm{q} j}$ einen Beitrag. Da der Integrand mit $\varepsilon^{\prime}$ nur langsam veränderlich und $\hbar \omega_{\mathrm{q}_{j}} \ll \zeta$ ist, begehen wir einen kleinen Fehler, wenn wir ihn an der Stelle $\varepsilon^{\prime}=\zeta$ nehmen.

Wie man aus den Gln. $\left(31\right.$ a - c) entnehmen kann, ist die Vektorfunktion $\mathfrak{J}\left(\mathfrak{f}, \mathfrak{f}^{\prime}\right)$ nur von dem Differenzvektor $\left(\mathfrak{f}^{\prime}-\mathfrak{f}\right)$ abhängig. Dieselbe Abhängigkeit besitzen auch die Frequenz $\omega_{\mathfrak{q}_{j}}$ und die Polarisation $\mathfrak{e}_{\mathfrak{q}_{j}}$, weil beide ihre Werte nicht ändern werden, wenn man zum Ausbreitungsvektor $\mathfrak{q}$ einen reziproken Gittervektor addiert. Der in der eckigen Klammer von

$$
\begin{array}{r}
M_{n l}^{m o}=\frac{1}{(2 \pi)^{5}} \frac{2 n^{2}}{\gamma_{0}} \frac{\hbar}{k T} \int\left[\sum_{j} \frac{\left|\left(\mathfrak{e}_{\mathrm{a} j} \cdot \mathfrak{I}\left(\mathfrak{k}, \mathfrak{k}^{\prime}\right)\right)\right|^{2}}{\left(\exp \left\{\hbar \omega_{\mathrm{a} j} / k T\right\}-1\right)\left(1-\exp \left\{-\hbar \omega_{\mathrm{a} j} / k T\right\}\right)}\right] \Pi_{n}^{m}(\cos \vartheta) e^{i m \varphi} \\
\left\{\Pi_{l}^{o}(\cos \vartheta) e^{i o \varphi}-\Pi_{l}^{o}\left(\cos \vartheta^{\prime}\right) e^{i o \varphi^{\prime}}\right\}\left(k^{2} \frac{\partial k}{\partial \varepsilon}\right)^{2} \mathrm{~d} \omega_{\mathrm{t}} \mathrm{d} \omega \mathrm{\ell}^{\prime}
\end{array}
$$

stehende Ausdruck ist daher nur eine Funktion des nichtreduzierten Ausbreitungsvektors $\mathfrak{p}=\mathfrak{1}-\mathfrak{l}^{\prime}$. In einem kubischen Kristall muß dieser Ausdruck invariant gegenüber allen Drehoperationen der Oktaedergruppe sein. Führen wir durch

$$
\mathfrak{p}=\mathfrak{H}-\mathfrak{l}^{\prime}=\left\{\begin{array}{l}
p_{x} \\
p_{y} \\
p_{z}
\end{array}\right\}=\left\{\begin{array}{c}
p \sin \bar{\vartheta} \cos \bar{\varphi} \\
p \sin \bar{\vartheta} \sin \bar{\varphi} \\
p \cos \bar{\vartheta}
\end{array}\right\}
$$

im $p$-Raum ein sphärisches Polarkoordinatensystem ein, dessen Polarachse in Richtung der kubischen Achse weist, dann können wir ihn in fol-

27 A. Sommerfeld u. H. Bethe, Handbuch der Physik (GeigerScheel) XXIV/2, Springer-Verlag 1933, Seite 528. gender Weise nach kubisch-sphärischen Harmonischen entwickeln

$$
\begin{gathered}
F(\mathfrak{p})=n^{2} \sum_{j} \frac{\mid\left(\left.e_{\mathrm{a} j} \Im\left(\mathfrak{f}, \mathfrak{p}^{\prime}\right)\right|^{2}\right.}{\left(\exp \left\{\hbar \omega_{\mathrm{q} j} / k T\right\}-1\right)\left(1-\exp \left\{\overline{\left.\left.-\hbar \omega_{\mathrm{a} j} / k T\right\}\right)}\right.\right.} \\
=\sum_{\varrho} F_{\varrho}(p) K_{2}(\bar{\vartheta}, \bar{\varphi})
\end{gathered}
$$

In der Näherung quasifreier Elektronen ist der Betrag des Vektors p gegeben durch

$$
p=k_{\mathrm{F}} \sqrt{2(1-\cos \Theta)},
$$

wenn $\Theta$ der Winkel zwischen $\mathfrak{f}^{\prime}$ und $\mathfrak{f}^{\prime}$ ist. Wir können daher außerdem noch die von $p$ abhängigen Koeffizienten $F_{\varrho}(p)$ nach Legendreschen Polynomen entwickeln

$$
F(\mathfrak{p})=\sum_{\varrho, \sigma} F_{\varrho \sigma} K_{2_{\ell}}(\bar{\vartheta}, \bar{\varphi}) P_{\sigma}(\cos \Theta) .
$$


Durch diesen Kunstgriff konnten wir in besonders einfacher Weise die Symmetrieeigenschaft des Kristalls berücksichtigen. Um die zur Berechnung des Matrixelementes $\underset{\substack{m o \\ n l \\ 00}}{\substack{n o \\ 0}}$ nötigen Integrationen durchführen zu können, müssen wir nun wieder die Variablen $\bar{\vartheta}, \bar{\varphi}$ und $\Theta$ durch $\vartheta, \vartheta^{\prime}, \varphi, \varphi^{\prime}$ ersetzen. Dies ist durch die aus Gl. (37) folgenden Substitution

$$
\begin{aligned}
\sin \bar{\vartheta} \cos \bar{\varphi} & =\frac{\sin \vartheta \cos \varphi-\sin \vartheta^{\prime} \cos \varphi^{\prime}}{\sqrt{2(1-\cos \Theta)}}, \\
\sin \bar{\vartheta} \sin \bar{\varphi} & =\frac{\sin \vartheta \sin \varphi-\sin \vartheta^{\prime} \sin \varphi^{\prime}}{\sqrt{2(1-\cos \Theta)}} \\
\cos \bar{\vartheta} & =\frac{\cos \vartheta-\cos \vartheta^{\prime}}{\sqrt{2(1-\cos \Theta)}},
\end{aligned}
$$

$\cos \Theta=\cos \vartheta \cos \vartheta^{\prime}+\sin \vartheta \sin \vartheta^{\prime} \cos \left(\varphi-\varphi^{\prime}\right)(41 \mathrm{~d})$ möglich. Im Anhang wird gezeigt, daß durch diese
Transformation der Ausdruck $P_{\sigma}(\cos \Theta) K_{2}(\bar{\vartheta}, \bar{\varphi})$ in eine nach einer Seite abbrechenden Reihe von bikubisch-sphärischen Harmonischen ${ }^{28}$ übergeht, die zur selben Darstellung $2 \varrho$ gehören

$$
\begin{aligned}
P_{\sigma}(\cos \Theta) K_{2}(\bar{\vartheta}, \bar{\varphi}) & (42) \\
& =\sum_{\nu, \lambda} k_{\nu \lambda}^{(2 \varrho ; \sigma,+)} B_{v \lambda}^{(2 \varrho,+)}\left(\vartheta, \varphi ; \vartheta^{\prime}, \varphi^{\prime}\right) .
\end{aligned}
$$

Setzen wir nun noch die Entwicklung der bikubisch-sphärischen Harmonischen nach Kugelfunktionsprodukten ein, dann können wir die restlichen Integrationen über $\vartheta, \vartheta^{\prime}, \varphi, \varphi^{\prime}$ ausführen, weil $\left(k^{2} \cdot \mathrm{d} k / \mathrm{d} \varepsilon\right)^{2}$ bei einem quasifreien Elektronengas winkelunabhängig ist. Nach kurzer Zwischenrechnung ergibt sich

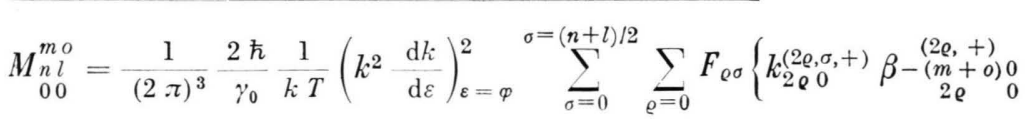

$$
\begin{aligned}
& \left.\cdot \sqrt{2} \int_{-1}^{1} \Pi_{2 \varrho}^{m+o}(x) \Pi_{n}^{m}(x) \Pi_{l}^{o}(x) \mathrm{d} x-k_{n l}^{(2 \varrho ; \sigma,+)} \beta \begin{array}{r}
(2 \rho,+) \\
n-o
\end{array}\right\} .
\end{aligned}
$$

Hierbei haben wir schon berücksichtigt, daß wegen des Vektoradditionsmodells nur die Funktion $\underset{2 \varrho 0}{B(2 \varrho,+)}\left(\vartheta, \varphi ; \vartheta^{\prime}, \varphi^{\prime}\right)$ von Null verschieden ist, wenn ein unterer Index Null ist. Während die Glieder in der geschweiften Klammer rein mathematische Größen sind, die sich ein für allemal mit Hilfe der Theorie der bikubisch-sphärischen Harmonischen berechnen lassen, wird mit den Entwicklungskoeffizienten $F_{\varrho \sigma}$ die physikalische Seite des Problems beschrieben.

\section{Berechnung des Gitterschwingungsspektrums mit Hilfe der Gitterdynamik}

Die Untersuchung von BAILys hat gezeigt, daß der elektrische Widerstand sehr stark von der Form

${ }^{28}$ Die bikubisch-sphärischen Harmonischen $B_{n l}^{(2 \varrho,+)}(\vartheta, \varphi$; $\left.\vartheta^{\prime}, \varphi^{\prime}\right)$ sind Funktionen der beiden Variablenpaare $\vartheta, \varphi$ und $\vartheta^{\prime}, \varphi^{\prime}$, die im selben Koordinatensystem definiert sind. Sie sind invariant gegenüber allen Symmetrieoperationen der Oktaedergruppe und lassen sich in folgender Weise nach Produkten von Kugelflächenfunktionen entwickeln

$$
\begin{array}{r}
B_{n l}^{(2 \varrho)}\left(\vartheta, \varphi ; \vartheta^{\prime}, \varphi^{\prime}\right)=\sum_{m, o} \underset{n l}{\beta \underset{m o}{(2 \varrho)}} \Pi_{n}^{m}(\cos \vartheta) \cdot \Pi_{l}^{o}\left(\cos \vartheta^{\prime}\right) \\
\cdot e^{i m \varphi \cdot e^{i o \varphi^{\prime}}}
\end{array}
$$

Die Koeffizienten $\underset{n l}{\beta m} \underset{n l}{(2 \varrho)}$ werden so festgelegt, da $\beta$ des Schwingungsspektrums abhängig sein kann, und daß es daher wünschenswert ist, das Spektrum mit Hilfe der Gittertheorie möglichst exakt zu berechnen. Im Gegensatz zu der elastischen Theorie, die seither bei der Berechnung des elektrischen Widerstandes benützt wurde, berücksichtigt man bei der Gittertheorie die atomistische Struktur des Kristalls, indem man zwischen den einzelnen Gitterbausteinen Kräfte annimmt, die bei der Verschiebung aus der Gleichgewichtslage auftreten. Durch die abschirmende Wirkung der Leitungselektronen nehmen diese Wechselwirkungskräfte sehr schnell $\mathrm{ab}$, so daß nur die Kopplung zwischen den nähergelegenen Gitterionen vorhanden ist. Im Rahmen einer allgemeinen Gittertheorie, wie sie von Bons und Mitarb. ${ }^{29-31}$ abgeleitet wurde, wird über die Form der Wechselwir-

$$
B_{n l}^{(2 \varrho)}\left(\vartheta, \varphi ; \vartheta^{\prime}, \varphi^{\prime}\right)
$$

zur Darstellung $D^{(2 \varrho)}$ der dreidimensionalen Drehgruppe gehört. Die Funktion $B_{n l}^{(2 \varrho,+)}\left(\vartheta, \varphi ; \vartheta^{\prime}, \varphi^{\prime}\right)$ ist außerdem invariant nüber der Vertauschung der beiden Variablenpaare $\vartheta, \varphi$ und $\vartheta^{\prime}, \varphi^{\prime}$. Eine weitere Untersuchung der Eigenschaften der bikubischen, sphärischen Harmonischen findet sich bei H. Bross, Z. Naturforschg., in Vorbereitung.

20 M. Born, Repts. Progr. Phys. 9, 356 [1942/43].

30 G. H. Begrie u. M. Born, Proc. Roy. Soc., Lond. A 188, 179 [1947].

31 G. H. Begrie, Proc. Roy. Soc., Lond. A 188, 189 [1947]. 
kungskräfte keine weitere Annahme gemacht. Da jedoch die potentielle Energie eines Kristalls invariant gegenüber Deckoperationen sein muß, kann man durch Symmetriebetrachtung die Anzahl der unabhängigen Kopplungskonstanten weitgehend herabsetzen. Wie BEGBIE ${ }^{31}$ z. B. gezeigt hat, gibt es bei einem kubisch-flächenzentrierten Kristall für die Charakterisierung der Wechselwirkung zwischen den nächsten Nachbarn 3 Kopplungskonstanten, zwischen übernächsten Nachbarn zwei und zwischen Nachbarn dritter Ordnung 4 Konstanten. Eine quantenmechanische Berechnung dieser Kopplungskonstanten ist bei den Edelmetallen wegen der Wirkung der dSchalen noch nicht gelungen ${ }^{32}$. Durch Untersuchung der diffusen Streuung von RönTgen-Strahlen, wie sie z. B. JACOBSEN ${ }^{33}$ für Kupfereinkristalle durchge- führt hat oder eventuell auch durch thermische Neutronenstreuung ${ }^{34}$, kann man jedoch die Kopplungskonstanten für nähergelegene Nachbarn auch experimentell bestimmen.

Mit diesen Kopplungskonstanten lassen sich nun die Eigenschwingungen eines Kristalls berechnen. Bei einem primitiven Gitter erhält man folgendes homogenes Gleichungssystem für die Amplitude einer fortschreitenden Welle, wenn wir der Bezeichnungsweise von LEIBFRIED ${ }^{35}$ folgen

$$
M \omega^{2} e=\mathbf{T}(p), e,
$$

dabei ist $M$ die Masse eines Gitterions, $\omega$ die Kreisfrequenz der Schallwelle mit Ausbreitungsvektor $\mathfrak{p}$; $|p|=2 \pi / \lambda$. Die Komponenten $T_{i j}(p)$ sind bei einem kubisch-flächenzentrierten Kristall gegeben durch

$$
\begin{aligned}
& T_{11}(\mathfrak{p})=4\left\{\begin{array}{c}
\left(a_{1}+2 b_{1}\right)+2\left(a_{3}+2 b_{3}\right)-\left(b_{1}+2 b_{3}\right)\left(C_{12}+C_{31}\right)-\left(a_{1}+2 a_{3}\right) C_{23} \\
\left.+\left(a_{2}+4 a_{3} C_{23}\right) S_{1}{ }^{2}+\left(b_{2}+4 b_{3} C_{31}\right) S_{2}{ }^{2}+\left(b_{2}+4 b_{3} C_{12}\right) S_{3}{ }^{2}\right\}
\end{array}\right. \\
& T_{12}(\mathfrak{p})=4 S_{1} S_{2}\left\{c_{1}+2 c_{2}-4 c_{3} S_{3}{ }^{2}+4 e_{3}\left(C_{13}+C_{32}\right)\right\}, \\
& C_{i j}=\cos \left(\frac{a_{0}}{2} p_{i}\right) \cos \left(\frac{a_{0}}{2} p_{j}\right),
\end{aligned}
$$

wenn man sich auf Nachbarn dritter Ordnung einschließlich beschränkt. Die übrigen Elemente von $\mathbf{T}(p)$ erhält man durch zyklische Vertauschung. $a_{0}$ ist die Gitterkonstante der kubisch-flächenzentrierten Zelle. Die Bezeichnungsweise der Kopplungskonstanten $a_{1}, b_{1}, c_{1}$ etc. wurde von JACOBSEN übernommen ${ }^{36}$.

Die Säkulargleichung der homogenen Gleichung

$$
\left|T_{i l}-\delta_{i l} M \omega^{2}\right|=0
$$

liefert zu jedem Ausbreitungsvektor $\mathfrak{p}$ drei Frequenzen $\omega_{j}$ und drei Vektoren $\mathrm{e}^{(j)}$, die die Polarisation

32 W. BreniG, Z. Phys. 142, 163 [1955].

33 E. H. Jacobsen, Phys. Rev. 97, 654 [1955].

34 B. N. Brockhouse u. A. T. Stewart, Rev. Mod. Phys. 30 , 236 [1958].

35 G. LeIbFried, Handbuch der Physik (Fü̈GGE) VII/1, Springer-Verlag, Berlin-Göttingen-Heidelberg 1955.

s6 Die von LeIBFried definierten Kopplungsmatrizen haben dann folgende Form:

$$
\begin{gathered}
\boldsymbol{\Phi}\left(a_{0} / 2\right)(0,1,1)=-\left\{\begin{array}{lll}
a_{1} & 0 & 0 \\
0 & b_{1} & c_{1} \\
0 & c_{1} & b_{1}
\end{array}\right\}, \\
\boldsymbol{\Phi}^{a_{0}(1,0,0)}=-\left\{\begin{array}{lll}
a_{2} & 0 & 0 \\
0 & b_{2} & 0 \\
0 & 0 & b_{2}
\end{array}\right\}, \\
\boldsymbol{\Phi}\left(a_{0} / 2\right)(2,1,1)=-\left\{\begin{array}{lll}
a_{3} & e_{3} & e_{3} \\
e_{3} & b_{3} & c_{3} \\
e_{3} & c_{3} & b_{3}
\end{array}\right\},
\end{gathered}
$$

$\boldsymbol{\Phi}^{(0,0,0)}=2\left(2 a_{1}+4 b_{1}+a_{2}+2 b_{2}+4 a_{3}+8 b_{3}\right) \cdot 1$. der Welle kennzeichnen. Diese drei Vektoren sind aufeinander orthogonal und können normiert werden

$$
\mathrm{e}^{(j)} \mathrm{e}^{\left(j^{\prime}\right)}=\delta_{j, j^{\prime}} .
$$

Im Gegensatz zu einer isotropen Theorie gibt es nur für besonders ausgezeichnete Richtungen des Ausbreitungsvektors $\mathfrak{p}$ einen Polarisationsvektor, der parallel zu $\mathfrak{p}$ ist (longitudinale Welle). Im allgemeinen hat das orthogonale Achsenkreuz der drei Polarisationsvektoren eine beliebige Orientierung zum Ausbreitungsvektor der Welle. Für die elektrische Leitfähigkeit und die Wärmeleitfähigkeit ist nur der Ausdruck $\left(p e^{(j)}\right)$ wichtig.

\section{Numerische Durchführung}

Von den einwertigen Metallen kann man zur Zeit nur bei Kupfer nähere Angaben über das Gitterschwingungsspektrum machen. Alle numerischen Untersuchungen haben wir daher an diesem Metall durchgeführt. Zunächst wurde mit Hilfe der neun von JACOBSEN angegebenen Kopplungsmatrizen die Dispersionskurve und die Polarisation für verschiedene Ausbreitungsrichtungen untersucht. Zur Berücksichtigung der Anisotropie des Gitterschwingungsspektrums haben wir eine Kugeloberfläche $p=$ const 
durch ein Netz von Punkten in nahezu gleich große Flächenstücke eingeteilt. Wegen der kubischen Symmetrie würde es genügen, wenn wir nur den 48-ten Teil einer Kugeloberfläche näher untersucht hätten. Für den weiteren Gang der Rechnung hat es sich als günstig erwiesen, die Hälfte eines Kugeloktanten mit den folgenden Ausbreitungsrichtungen zu benützen :

\begin{tabular}{|l|c|c|c|}
\hline $\cos \vartheta$ & $\bar{\varphi}=0$ & $\pi / 8$ & $\pi / 4$ \\
\hline 0 & 6 & 24 & 12 \\
0,25 & 24 & 48 & 24 \\
0,50 & 24 & 48 & 24 \\
0,75 & 24 & 48 & 24 \\
\hline
\end{tabular}

In der Tabelle haben wir ferner angegeben, wie oft der betreffende Funktionswert infolge der kubischen Symmetrie auf der ganzen Kugeloberfläche vorkommt (Gesamtzahl 330 Richtungen). Wir glauben, daß die so ausgewählten Richtungen genügen, um die anisotropen Effekte im Kristall im wesentlichen richtig wiederzugeben. Es ist wahrscheinlich wenig sinnvoll, wesentlich mehr Ausbreitungsrichtungen in Betracht zu ziehen, weil die experimentell gemessenen Kopplungskonstanten gar nicht so genau bekannt sind ${ }^{37}$.

Wie schon früher erwähnt wurde, ist der Vektor $p$ kein reduzierter Ausbreitungsvektor. Als Differenzvektor der Elektronenausbreitungsvektoren $\mathfrak{f}^{\prime}$ und $\mathfrak{f}^{\prime}$ kann sein Betrag zwischen 0 (Vorwärtsstreuung) und $2 k_{\mathrm{F}}$ (Rückwärtsstreuung) liegen. Wegen

$k_{\mathrm{F}}=\left(3 \pi^{2} \cdot 4 / a_{0}{ }^{3}\right)^{1 / 3} \quad$ ist $\quad p_{\max }=2(3 / 16 \pi)^{1 / 3} B$

$(B$ ist die Gitterkonstante der reziproken Elementarzelle). Um die nachfolgende Integration besser durchführen zu können, legen wir den Betrag von $\mathfrak{p}$ nach Gl. (39) durch den Winkel $\Theta$ zwischen $\mathfrak{f}^{\prime}$ und $\mathfrak{f}^{\prime}$ fest, und zwar nehmen wir für $\cos \Theta$ folgende Werte an:

\begin{tabular}{|c|c|c|c|c|c|c|c|c|}
\hline $\cos \theta$ & 1,0 & 0,8 & 0,6 & 0,2 & $-0,2$ & $-0,6$ & $-0,8$ & $-1,0$ \\
\hline$p / B$ & 0 & 0,2472 & 0,3495 & 0,4943 & 0,6054 & $\overline{0,6991}$ & 0,7415 & 0,7816 \\
\hline
\end{tabular}

Die für die verschiedenen Ausbreitungsrichtungen berechnete Kreisfrequenz $\omega_{\mathfrak{p} j}$ und das allein für die elektrische Leitfähigkeit wichtige Skalarprodukt $\left(\mathfrak{e}^{(j)} p^{0}\right)$ sind als Funktion des Ausbreitungsvektors in Abb. 2 dargestellt.

37 Eine nähere Untersuchung der Genauigkeit der Kopplungsmatrizen wurde von C. B. WALKER, Phys. Rev. 103, 547 [1956] durchgeführt.

38 K. Fuchs, Proc. Roy. Soc., Lond. A 151, 585 [1935].

39 K. Kambe, Phys. Rev. 99, 419 [1955]. freien Elektronengas im wesentlichen durch

$$
\begin{gathered}
F(\mathfrak{p})=n^{2} \sum_{j} \frac{\left|\left(\mathfrak{e}_{\mathfrak{p}}^{(j)} \cdot \mathfrak{I}\left(\mathfrak{k}, \mathfrak{k}^{\prime}\right)\right)\right|^{2}}{\left(\exp \left\{\hbar \omega \mathfrak{p}_{j} / k T\right\}-1\right)\left(1-\exp \left\{-\hbar \omega \mathfrak{p}_{j} / k T\right\}\right)} \\
=|n J(p)|^{2} \frac{\sum}{j} \frac{\left(\mathfrak{e}_{\mathfrak{p}}^{(j)} \cdot p^{0}\right)^{2}}{4 \cdot \Im_{\operatorname{in}^{2}} \frac{\hbar \omega \mathfrak{p} j}{2 k T}}
\end{gathered}
$$

Der elektrische Widerstand ist bei einem quasibestimmt; dabei ist nach Gl. (31 a) der Betrag des Vektors $\left|\mathfrak{\Im}\left(\mathfrak{f}, \mathfrak{f}^{\prime}\right)\right|=J(p)$ nur eine Funktion des Winkels $\Theta$. Die Anisotropie des Streumechanis. mus wird also nur durch den hinter dem Summationszeichen stehenden Ausdruck beschrieben. Im Gegensatz zu einer Kontinuumstheorie, wo wegen des linearen Zusammenhangs zwischen Frequenz und Ausbreitungsvektor die Temperaturabhängigkeit des elektrischen Widerstandes in geschlossener Form angegeben werden kann, können wir ihn nur für bestimmte Werte berechnen, und zwar haben wir die folgenden Temperaturen gewählt: $T=20,4{ }^{\circ} \mathrm{K}$, $T=77,4{ }^{\circ} \mathrm{K}$ und $T=273,4{ }^{\circ} \mathrm{K}$.

Die numerische Auswertung ergibt, daß bei tiefen Temperaturen für einen bestimmten Ausbreitungsvektor $p$ die Gitterschwingungen mit kleinster Frequenz, die meist nahezu transversalen Charakter besitzen, den Hauptbeitrag zur Streuung liefern. Bei höheren Temperaturen haben dagegen die Schwingungen mit mehr longitudinalem Charakter die größere Bedeutung. Bei der numerischen Berechnung von $J(p)$ tritt die Schwierigkeit auf, daß der Wert $\left[U_{0}{ }^{\prime}\left(r_{\mathrm{s}}\right)-E_{0}{ }^{\prime}\right]$ und auch die FERMI-Energie nicht genau bekannt sind. Während aus der Arbeit von Fuchs ${ }^{38}$ für $\left[U_{0}{ }^{\prime}\left(r_{\mathrm{s}}\right)-E_{0}{ }^{\prime}\right]$ der Wert $1,3 \mathrm{eV}$ zu entnehmen ist, erhält man aus den Angaben von $\mathrm{K}_{\text {AMBE }}{ }^{39}$ gerade den doppelten Wert. Wir haben daher die ganze Rechnung mit den folgenden Parametern durchgeführt:

$\left[U_{0}{ }^{\prime}\left(r_{\mathrm{s}}\right)-E_{0}{ }^{\prime}\right] / \zeta=0,0,185,0,370$ und für die Fermi-Energie $7,0 \mathrm{eV} \quad\left(m^{*} / m=1\right) \quad$ bzw. $4,7 \mathrm{eV}$ $\left(m^{*} / m=1,5\right)$.

Für die Entwicklung des Ausdrucks $F(p)$ nach kubisch-sphärischen Harmonischen $K_{2 \varrho}(\vartheta, \bar{\varphi})$ benützen wir die Methode der kleinsten Quadrate: Als Vergleichsfunktion dient die Entwicklung

$$
\sum_{\varrho=0}^{4} F_{\varrho}(p) K_{2}(\bar{\vartheta}, \bar{\varphi}),
$$

deren Koeffizienten aus der Forderung bestimmt werden, daß die Summe der Abweichungsquadrate 
$\boldsymbol{T}=\mathbf{2 0 , 4 ^ { \circ } \mathrm { K } :}$

$4 \pi^{3} \frac{\gamma_{0}}{\hbar} \frac{k T}{(\zeta B)^{2}}\left(k^{2} \frac{\mathrm{d} k}{\mathrm{~d} \varepsilon}\right)^{-2} \cdot M_{n=0}^{m o} \cdot 10^{6}$

\begin{tabular}{|c|c|c|c|c|c|c|c|}
\hline \multirow{2}{*}{\multicolumn{2}{|c|}{$\left[V_{\mathrm{o}}\left(r_{\mathrm{s}}\right)-E_{0}\right] / \zeta$}} & \multicolumn{3}{|c|}{$m^{*} / m=1,0$} & \multicolumn{3}{|c|}{$m^{*} / m=1,5$} \\
\hline & & 0,000 & 0,185 & 0,370 & 0,000 & 0,185 & 0,370 \\
\hline $\begin{array}{c}n l \\
11\end{array}$ & $\begin{array}{rr}m & 0 \\
0 & 0 \\
1 & -1\end{array}$ & $\begin{array}{r}0,4045 \\
-0,4045\end{array}$ & $\begin{array}{r}0,8122 \\
-0,8122\end{array}$ & $\begin{array}{r}1,4165 \\
-1,4165\end{array}$ & $\begin{array}{r}0,6166 \\
-0,6166\end{array}$ & $\begin{array}{r}1,1045 \\
-1,1045\end{array}$ & $\begin{array}{r}1,7446 \\
-1,7446\end{array}$ \\
\hline 13 & $\begin{array}{lr}0 & 0 \\
1 & -1 \\
1 & 3\end{array}$ & $\begin{array}{l}-0,1522 \\
-0,0933 \\
-0,1204\end{array}$ & $\begin{array}{l}-0,3492 \\
-0,2138 \\
-0,2761\end{array}$ & $\begin{array}{l}-0,6367 \\
-0,3899 \\
-0,5034\end{array}$ & $\begin{array}{l}-0,2550 \\
-0,1562 \\
-0,2016\end{array}$ & $\begin{array}{l}-0,4851 \\
-0,2970 \\
-0,3835\end{array}$ & $\begin{array}{l}-0,7906 \\
-0,4841 \\
-0,6250\end{array}$ \\
\hline 33 & $\begin{array}{lr}0 & 0 \\
1 & -1 \\
2 & -2 \\
3 & -3 \\
1 & 3 \\
2 & 2\end{array}$ & $\begin{array}{r}0,6492 \\
-0,7089 \\
0,8044 \\
-0,6850 \\
0,0462 \\
-0,0597\end{array}$ & $\begin{array}{r}0,7508 \\
-0,8814 \\
1,0905 \\
-0,8292 \\
0,1012 \\
-0,1307\end{array}$ & $\begin{array}{r}0,9233 \\
-1,1593 \\
1,5369 \\
-1,0649 \\
0,1828 \\
-0,2360\end{array}$ & $\begin{array}{r}0,5753 \\
-0,6680 \\
0,8163 \\
-0,6309 \\
0,0718 \\
-0,0927\end{array}$ & $\begin{array}{r}0,8158 \\
-0,9950 \\
1,2818 \\
-0,9233 \\
0,1388 \\
-0,1792\end{array}$ & $\begin{array}{r}1,1259 \\
-1,4212 \\
1,8935 \\
-1,3031 \\
0,2287 \\
-0,2952\end{array}$ \\
\hline
\end{tabular}

$\boldsymbol{T}=77,4^{\circ} \mathrm{K}: \quad 4 \pi^{3} \frac{\gamma_{0}}{\hbar} \frac{k T}{(\zeta B)^{2}}\left(k^{2} \frac{\mathrm{d} k}{\mathrm{~d} \varepsilon}\right)^{-2} \underset{00}{M n l} \operatorname{mo}_{00}^{m l} \cdot 10^{3}$

\begin{tabular}{|c|c|c|c|c|c|c|c|}
\hline \multirow{2}{*}{\multicolumn{2}{|c|}{$\left[V_{\mathrm{o}}\left(r_{\mathrm{s}}\right)-E_{0}\right] / \zeta$}} & \multicolumn{3}{|c|}{$m^{*} / m=1,0$} & \multicolumn{3}{|c|}{$m^{*} / m=1,5$} \\
\hline & & 0,000 & 0,185 & 0,370 & 0,000 & 0,185 & 0,370 \\
\hline $\begin{array}{l}n l \\
11\end{array}$ & $\begin{array}{cr}m & o \\
0 & 0 \\
1 & -1\end{array}$ & $\begin{array}{r}0,3846 \\
-0,3846\end{array}$ & $\begin{array}{r}0,6946 \\
-0,6946\end{array}$ & $\begin{array}{r}1,1112 \\
-1,1112\end{array}$ & $\begin{array}{r}0,5924 \\
-0,5924\end{array}$ & $\begin{array}{r}0,9146 \\
-0,9146\end{array}$ & $\begin{array}{r}1,3176 \\
-1,3176\end{array}$ \\
\hline 13 & $\begin{array}{lr}0 & 0 \\
1 & -1 \\
1 & 3\end{array}$ & $\begin{array}{l}-0,0223 \\
-0,0136 \\
-0,0176\end{array}$ & $\begin{array}{l}-0,0401 \\
-0,0246 \\
-0,0317\end{array}$ & $\begin{array}{l}-0,0649 \\
-0,0398 \\
-0,0513\end{array}$ & $\begin{array}{l}-0,0336 \\
-0,0206 \\
-0,0266\end{array}$ & $\begin{array}{l}-0,0529 \\
-0,0324 \\
-0,0418\end{array}$ & $\begin{array}{l}-0,0776 \\
-0,0475 \\
-0,0613\end{array}$ \\
\hline 33 & $\begin{array}{lr}0 & 0 \\
1-1 \\
2-2 \\
3-3 \\
1 & 3 \\
1 & 2 \\
2 & 2\end{array}$ & $\begin{array}{r}0,5677 \\
-0,5832 \\
0,6079 \\
-0,5770 \\
0,0120 \\
-0,0155\end{array}$ & $\begin{array}{r}0,8345 \\
-0,8586 \\
0,8971 \\
-0,8490 \\
0,0186 \\
-0,0241\end{array}$ & $\begin{array}{r}1,1759 \\
-1,2113 \\
1,2680 \\
-1,1971 \\
0,0275 \\
-0,0355\end{array}$ & $\begin{array}{r}0,7691 \\
-0,7907 \\
0,8253 \\
-0,7821 \\
0,0167 \\
-0,0216\end{array}$ & $\begin{array}{r}1,0303 \\
-1,0607 \\
1,1093 \\
-1,0485 \\
0,0235 \\
-0,0304\end{array}$ & $\begin{array}{r}1,3458 \\
-1,3871 \\
1,4531 \\
-1,3706 \\
0,0320 \\
-0,0413\end{array}$ \\
\hline
\end{tabular}

$\boldsymbol{T}>\Theta:$

$4 \pi^{3} \frac{\gamma_{0}}{\hbar} \frac{k T}{(\zeta B)^{2}}\left(k^{2} \frac{\mathrm{d} k}{\mathrm{~d} \varepsilon}\right)^{-2} \cdot\left(\frac{\hbar \cdot 10^{13}}{k T}\right)^{+2} \cdot \underset{00}{M_{n l} o} \cdot 10^{3}$

\begin{tabular}{|c|c|c|c|c|c|c|c|}
\hline \multirow{2}{*}{\multicolumn{2}{|c|}{$\left[V_{\mathrm{o}}\left(r_{\mathrm{s}}\right)-E_{0}\right] / \zeta$}} & \multicolumn{3}{|c|}{$m^{*} / m=1,0$} & \multicolumn{3}{|c|}{$m^{*} / m=1,5$} \\
\hline & & 0,000 & 0,185 & 0,370 & 0,000 & 0,185 & 0,370 \\
\hline $\begin{array}{l}n l \\
11\end{array}$ & $\begin{array}{rr}m & 0 \\
0 & 0 \\
1 & -1\end{array}$ & $\begin{array}{r}0,8244 \\
-0,8244\end{array}$ & $\begin{array}{r}1,4382 \\
-1,4382\end{array}$ & $\begin{array}{r}2,2514 \\
-2,2514\end{array}$ & $\begin{array}{r}1,2496 \\
-1,2496\end{array}$ & $\begin{array}{r}1,8771 \\
-1,8771\end{array}$ & $\begin{array}{r}2,6531 \\
-2,6531\end{array}$ \\
\hline 13 & $\begin{array}{lr}0 & 0 \\
1 & -1 \\
1 & 3\end{array}$ & $\begin{array}{l}-0,0278 \\
-0,0171 \\
-0,0222\end{array}$ & $\begin{array}{l}-0,0482 \\
-0,0295 \\
-0,0381\end{array}$ & $\begin{array}{l}-0,0762 \\
-0,0466 \\
-0,0602\end{array}$ & $\begin{array}{l}-0,0412 \\
-0,0264 \\
-0,0325\end{array}$ & $\begin{array}{l}-0,0628 \\
-0,0388 \\
-0,0496\end{array}$ & $\begin{array}{l}-0,0904 \\
-0,0554 \\
-0,0714\end{array}$ \\
\hline 33 & $\begin{array}{lr}0 & 0 \\
1-1 \\
2-2 \\
3-3 \\
1 & 3 \\
2 & 2\end{array}$ & $\begin{array}{r}1,2854 \\
-1,3068 \\
1,3409 \\
-1,2982 \\
0,0166 \\
-0,0213\end{array}$ & $\begin{array}{r}1,8795 \\
-1,9119 \\
1,9639 \\
-1,8989 \\
0,0251 \\
-0,0325\end{array}$ & $\begin{array}{r}2,6261 \\
-2,6729 \\
2,7479 \\
-2,6542 \\
0,0363 \\
-0,0468\end{array}$ & $\begin{array}{r}1,7498 \\
-1,7794 \\
1,8267 \\
-1,7675 \\
0,0229 \\
-0,0296\end{array}$ & $\begin{array}{r}2,3194 \\
-2,3600 \\
2,4250 \\
-2,3437 \\
0,0315 \\
-0,0406\end{array}$ & $\begin{array}{r}2,9972 \\
-3,0514 \\
3,1382 \\
-3,0298 \\
0,0419 \\
-0,0542\end{array}$ \\
\hline
\end{tabular}

Tab. 1. Matrixelemente $M$ bei verschiedenen Temperaturen. 
für die 330 Raumrichtungen zu einem Minimum wird. Das Ergebnis dieser Entwicklung für

$$
\left[U_{0}{ }^{\prime}\left(r_{\mathrm{s}}\right)-E_{0}{ }^{\prime}\right] / \zeta=0,185 \text { und } \zeta=4,65 \mathrm{eV}
$$

findet sich für verschiedene Temperaturen in Abb. 3 , 4 und 5. Die Berechnung von $F_{\varrho, \sigma}$, d. h. die Entwicklung von $F_{\varrho}(p)$ nach Legendreschen Polynomen wird mit dem von FehlberG ${ }^{40}$ angegebenen Verfahren durchgeführt. Mit den so berechneten Koeffizienten lassen sich die Matrixelemente $\begin{gathered}m i \\ n l \\ r s\end{gathered}$ (Tab. 1) und damit auch der Widerstandstensor berechnen.

\section{Allgemeine Diskussion; Abweichungen von der Matthiessenschen Regel}

\section{a) Die elektrische Leitfähigkeit}

Aus den Abb. 3, 4 und 5 ist ersichtlich, daß die seitherige Annahme einer isotropen Übergangswahrscheinlichkeit für die thermische Streuung der Elektronen eine sehr grobe Annäherung an die wirklichen phvsikalischen Verhältnisse ist. Während in der üblichen Transporttheorie, wo man entweder das Gitterschwingungsspektrum mit der DeByeschen Theorie berechnet oder aber über das anisotrope Gitterspektrum (BAILYN) mittelt, nur der Entwicklungskoeffizient $F_{0}(p)$ den ganzen Streuprozeß beschreibt, sind in einer genaueren Theorie auch verschiedene Koeffizienten $F_{o}(p)$, wobei $o \neq 0$ ist, mit dem „isotropen“ Ausdruck $F_{0}(p)$ größenordnungsmäßig vergleichbar. Vor allem bei tiefer Temperatur ist diese Anisotropie sehr ausgeprägt. Neben den Gitterschwingungen mit kleinem Ausbreitungsvektor (Vorwärtsstreuung) sind hier vor allem nur noch jene Schwingungen mit großem Ausbreitungsvektor angeregt, die sich in [111]-Richtung ausbreiten. In dieser Richtung kommt nämlich die Kugel $p_{\max }=2 k_{\mathrm{F}}$ einigen reziproken Gitterpunkten am nächsten. Durch diese Umklappprozesse nimmt auch die Funktion $F_{0}(p)$ mit wachsendem $p$ wieder zu, während sie in der Blochschen Theorie nur in der Umgebung von $p=0 \mathrm{im}$ wesentlichen von Null verschieden ist. Bei höheren Temperaturen geht die Anisotropie immer mehr zurück, weil dann nicht nur die Gitterschwingungen für bestimmte Ausbreitungsrichtungen angeregt sind.

Um die theoretischen Ergebnisse mit dem Experi-

40 E. Fehlberg, Z. angew. Math. Mech. 104, 31 [1951].

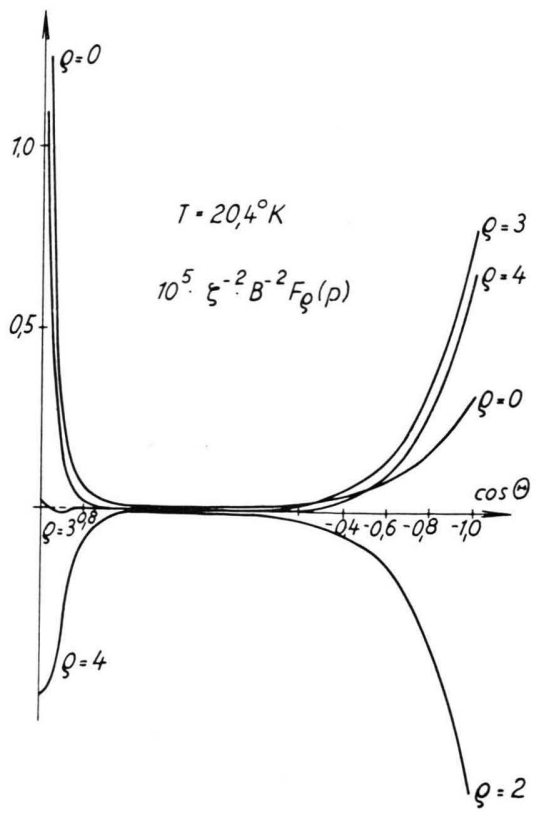

Abb. 3. Einfluß der Anisotropie des Gitterschwingungsspektrums auf die Elektron-Phonon-Streuung bei $T=20,4^{\circ} \mathrm{K}$.

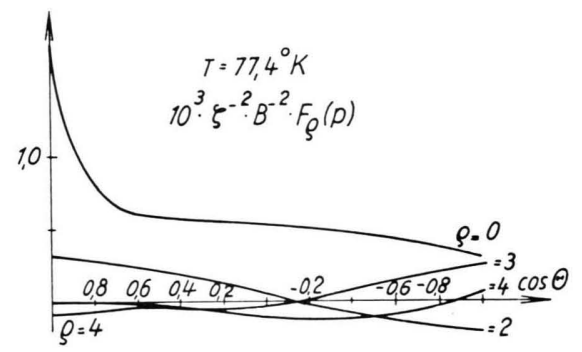

Abb. 4. Einfluß der Anisotropie des Gitterschwingungsspektrums auf die Elektron-Phonon-Streuung bei $T=77,4^{\circ} \mathrm{K}$.

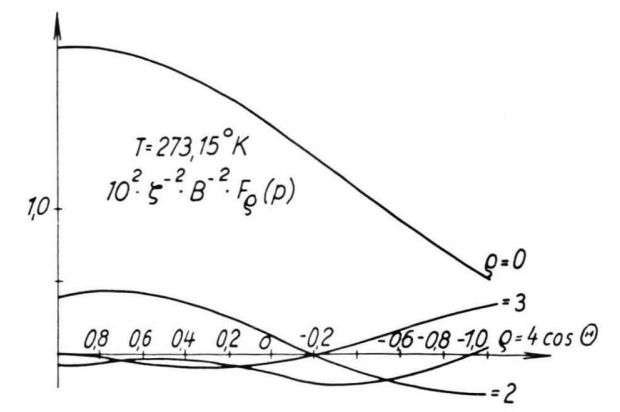

Abb. 5. Einfluß der Anisotropie des Gitterschwingungsspektrums auf die Elektron-Phonon-Streuung bei $T=273,15^{\circ} \mathrm{K}$.

ment vergleichen zu können, müssen wir noch für die Parameter $\zeta$ und $U_{0}{ }^{\prime}\left(r_{\mathrm{s}}\right)-E_{0}{ }^{\prime}$ Zahlenwerte einsetzen. Zunächst sieht man aus Tab. 2, daß das Verhältnis $\varrho_{T}^{(0)} / \varrho_{T_{0}}^{(0)}$, wo $\varrho_{T_{0}}^{(0)}$ der Widerstand bei 


$$
\boldsymbol{T}=\mathbf{2 0 , 4 ^ { \circ } \mathrm { K }}
$$

\begin{tabular}{|c|c|c|c|c|c|c|}
\hline & \multicolumn{3}{|c|}{$m^{*} / m=1,0$} & \multicolumn{3}{c|}{$m^{*} / m=1,5$} \\
\hline$\left[U_{0}\left(r_{\mathrm{s}}\right)-E_{0}\right] / \zeta$ & 0,000 & 0,185 & 0,370 & 0,000 & 0,185 & 0,370 \\
$\varrho_{T}^{(0)} / \varrho_{T_{\mathrm{\bullet}}}^{(0)} \cdot 10^{4}$ & 4,67 & 4,73 & 4,55 & 4,22 & 4,56 & 4,69 \\
$a_{T} / a_{T_{0}}$ & 0,918 & 0,676 & 0,287 & 0,705 & 0,470 & 0,260 \\
$10^{6} \cdot b\left(\mathrm{Oe}^{-2}\right)$ & 3,030 & 5,496 & 6,190 & 8,487 & 6,384 & 4,309 \\
$10^{6} \cdot c\left(\mathrm{Oe}^{-2}\right)$ & 0,056 & 0,724 & 0,519 & 1,187 & 0,667 & 0,345 \\
$10^{6} \cdot A_{\|}\left(\mathrm{Oe}^{-2}\right)$ & 1,436 & 2,488 & 2,684 & 3,870 & 2,820 & 1,862 \\
$10^{6} \cdot A_{\perp}\left(\mathrm{Oe}^{-2}\right)$ & 2,313 & 4,252 & 4,848 & 6,552 & 4,974 & 3,378 \\
\hline
\end{tabular}

$$
\boldsymbol{T}=77,4^{\circ} \mathrm{K}
$$

\begin{tabular}{|c|c|c|c|c|c|c|}
\hline & \multicolumn{3}{|c|}{$m^{*} / m=1,0$} & \multicolumn{3}{c|}{$m^{*} / m=1,5$} \\
\hline$\left[U_{0}\left(r_{\mathrm{s}}\right)-E_{0}\right] / \zeta$ & 0,000 & 0,185 & 0,370 & 0,000 & 0,185 & 0,370 \\
$\varrho_{T}^{(0)} / \varrho_{T_{0}}^{(0)}$ & 0,129 & 0,133 & 0,136 & 0,131 & 0,134 & 0,137 \\
$a_{T} / a_{T_{0}}$ & 0,997 & 0,996 & 0,995 & 0,997 & 0,996 & 0,995 \\
$10^{12} \cdot b\left(\mathrm{Oe}^{-2}\right)$ & 1,418 & 0,804 & 0,470 & 0,844 & 0,562 & 0,377 \\
$10^{12} \cdot c\left(\mathrm{Oe}^{-2}\right)$ & 0,326 & 0,184 & 0,107 & 0,194 & 0,129 & 0,086 \\
$10^{12} \cdot \mathrm{A}_{\mid l}\left(\mathrm{Oe}^{-2}\right)$ & 0,697 & 0,395 & 0,231 & 0,415 & 0,276 & 0,185 \\
$10^{12} \cdot A_{\perp}\left(\mathrm{Oe}^{-2}\right)$ & 1,069 & 0,606 & 0,354 & 0,637 & 0,424 & 0,284 \\
\hline
\end{tabular}

$$
\boldsymbol{T}=\mathbf{2 7 3 , 1 5}{ }^{\circ} \mathrm{K}
$$

\begin{tabular}{|c|c|c|c|c|c|c|}
\hline & \multicolumn{3}{|c|}{$m^{*} / m=1,0$} & \multicolumn{3}{c|}{$m^{*} / m=1,5$} \\
\hline$\left[U_{0}\left(r_{\mathrm{s}}\right)-E_{0}\right] / \zeta$ & 0,000 & 0,185 & 0,370 & 0,000 & 0,185 & 0,370 \\
$\varrho_{T}^{(0)}[\mu \Omega \mathrm{cm}]$ & 0,376 & 0,655 & 1,026 & 0,570 & 0,856 \\
$\varrho_{T_{0}^{(0)}}^{(0)} a_{T_{0}}\left[\Omega \mathrm{cm} \mathrm{Oe}^{-1}\right]$ & & \multicolumn{2}{|c|}{$\mathbf{7 , 2 0 9}$} \\
$10^{14} \cdot b\left(\mathrm{Oe}^{-2}\right)$ & 0,681 & 0,375 & 0,220 & 0,394 & 0,259 & 0,177 \\
$10^{14} \cdot c\left(\mathrm{Oe}^{-2}\right)$ & 0,162 & 0,089 & 0,052 & 0,094 & 0,061 & 0,042 \\
$10^{14} \cdot A_{\|}\left(\mathrm{Oe}^{-2}\right)$ & 0,337 & 0,185 & 0,109 & 0,195 & 0,128 & 0,088 \\
$10^{14} \cdot A_{\perp}\left(\mathrm{Oe}^{-2}\right)$ & 0,512 & 0,282 & 0,166 & 0,297 & 0,195 & 0,133 \\
\hline
\end{tabular}

Tab. 2. Elektrischer Widerstand, Hall-Konstante und Änderungen des elektrischen Widerstandes im Magnetf $\in$ ld bei $T=20,4{ }^{\circ} \mathrm{K}, 77,4{ }^{\circ} \mathrm{K}$ und $273,15^{\circ} \mathrm{K}$.

$273,15^{\circ} \mathrm{K}$ ist, nur sehr wenig von diesen beiden Größen abhängig ist. Es genügt daher, diese Abhängigkeit bei $T_{0}=273,15^{\circ} \mathrm{K}$ zu untersuchen, was in Abb. 6 dargestellt ist. Legen wir in $U_{0}{ }^{\prime}\left(r_{\mathrm{s}}\right)-E_{0}{ }^{\prime}$ den von $\mathrm{K}_{\mathrm{AmBE}}{ }^{39}$ berechneten Wert von $E_{0}=-1,0937$ Rydberg zugrunde, dann erhält man bei einem quasifreien Elektronengas

$$
U_{0}{ }^{\prime}\left(r_{\mathrm{s}}\right)-E_{0}{ }^{\prime}=2,65 \mathrm{eV} \text {. }
$$

Für den spezifischen Widerstand bei $T=273,15^{\circ} \mathrm{K}$ können wir dann aus Abb. 6 die Werte $1,64 \mu \Omega \mathrm{cm}$ bzw. 1,02 $\mu \Omega$ cm entnehmen, je nachdem $m^{*} / m=1,5$ bzw. $m^{*} / m=1,0$ ist. Der experimentell gemessene Wert ist $1,57 \mu \Omega \mathrm{cm}$ und führt bei Annahme einer linearen Abhängigkeit von der effektiven Masse auf $m^{*} / m=1,44$, was in guter Übereinstimmung mit jenem Wert der effektiven Masse ist, der von

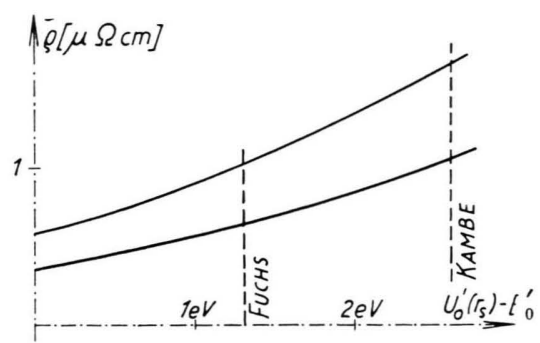

Abb. 6. Spezifischer Widerstand in Abhängigkeit von $U_{0}^{\prime}\left(r_{\mathrm{s}}\right)-E_{0}^{\prime}$. 
RAYNE $^{41}$ aus der spezifischen Wärme der Elektronen gemessen wurde. Mit dem von Fuchs berechneten Wert für $E_{0}$ erhält man einen zu kleinen Wert für den elektrischen Widerstand bei Zimmertemperatur.

Nachdem der Absolutwert des elektrischen Widerstandes bei $T=273,15^{\circ} \mathrm{K}$ festgelegt ist, brauchen wir nur noch das Verhältnis $\varrho_{T}^{(0)} / \varrho_{T_{0}}^{(0)}$ näher zu untersuchen. Die in der Tab. 2 aufgeführten Widerstandsverhältnisse für die Temperaturen $T=77,4{ }^{\circ} \mathrm{K}$ und $T=20,4{ }^{\circ} \mathrm{K}$ sind mit den von GrüNEISEN ${ }^{42}$ und Meissner ${ }^{43}$ experimentell ermittelten Werten von 0,129 bzw. $5 \cdot 10^{-4}$ zu vergleichen ${ }^{44}$. Wir erhalten für die Temperaturabhängigkeit im Intervall $20,4{ }^{\circ} \mathrm{K}<T<273,15^{\circ} \mathrm{K}$ gute Übereinstimmung zwischen Theorie und Experiment. In der Blochschen Theorie war dies auch der Fall; aber es ist doch zu berücksichtigen, daß dort der Wert der Debye-Temperatur $\Theta_{\mathrm{R}}$ den experimentell gemessenen Widerstandsverhältnissen angepaßt wurde. Da in der Blochschen Theorie der Widerstand bei tiefen Temperaturen eine Funktion von $\Theta_{R}{ }^{6}$ ist, kann diese Anpassung schon durch kleine Änderungen der Debye-Temperatur erreicht werden.

Auf eine Berechnung des elektrischen Widerstandes unterhalb $20{ }^{\circ} \mathrm{K}$ haben wir verzichtet, weil dann wahrscheinlich die Annahme, daß die Schallschwingungen im thermischen Gleichgewicht sind, nicht mehr erfüllt ist. Außerdem ist auch der temperaturabhängige Widerstand in diesem Bereich nicht mehr von großem Interesse, weil er meist vom Restwiderstand überdeckt wird.

\section{b) Abweichungen von der Matthiessenschen Regel}

Der Vorteil des oben beschriebenen Lösungsverfahrens gegenüber der Blochschen Methode erweist sich erst, wenn wir die Abweichungen von der Additivität des temperaturabhängigen Widerstandes und des Restwiderstandes untersuchen wollen. Nimmt man nämlich an, daß die thermische Streuung der Elektronen isotrop erfolgt, so ist nur dann eine Abweichung von der Matthiessenschen Regel zu erwarten, wenn die statischen Gitterfehler anisotrop streuen. Anders dagegen, wenn auch die ther-

41 J. Rayne, Phys. Rev. 95, 1428 [1954].

42 E. Grüneisen u. E. Goens, Z. Phys. 44, 615 [1927].

43 W. Meissner, Phys. Z. 29, 897 [1928].

44 Aus den Messungen von K. Berman und D.K.C. MacDonald, Proc. Roy. Soc., Lond. A 211, 122 [1954] kann man für das Widerstandsverhältnis $\varrho_{T}^{(0)} / \varrho_{T_{0}}^{(0)}$ bei $20,4{ }^{\circ} \mathrm{K}$ den Wert $5,96 \cdot 10^{-4}$ entnehmen. mischen Schwingungen die Elektronen anisotrop streuen! Dann können nämlich Abweichungen von der Additivität des Widerstandes auch durch isotrop streuende Gitterfehler hervorgerufen werden. Um dies zu zeigen, knüpfen wir an frühere Untersuchungen ${ }^{12}$ an. Wie dort schon erwähnt wurde, setzen sich die Matrixelemente additiv aus zwei Größen zusammen, wenn die Elektronen außerdem noch durch statische Gitterfehler gestreut werden:

$$
M_{n l}^{m o}=M_{\mathrm{t}} \underset{r s}{m o}+M_{\mathrm{R}} \underset{r s}{n l} \begin{aligned}
& m o \\
& r s
\end{aligned},
$$

wobei das Glied $M_{\mathrm{t}} \underset{r s}{m o} \begin{aligned} & m o \\ & r s\end{aligned}$ von der Streuung der Elektronen durch Gitterschwingungen, das Glied $M_{\mathrm{R}} \begin{gathered}m o \\ n l \\ r s\end{gathered}$ durch die Streuung an den statischen Gitterfehlern hervorgerufen wird. Wenn auch die statischen Gitterfehler kubische Symmetrie haben - ohne viel Schwierigkeiten kann man die folgenden Überlegungen jedoch auch auf tetragonale Symmetrie übertragen - ist der elektrische Widerstand gegeben durch

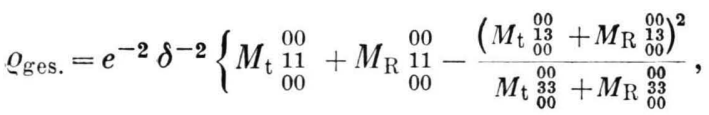

während sich der Widerstand $\varrho_{t}$, der durch die thermische Bewegung der Gitterionen, und der Widerstand $\varrho_{\mathrm{R}}$, der durch die statischen Gitterfehler erzeugt wird (Restwiderstand), aus den beiden folgenden Gleichungen berechnen lassen

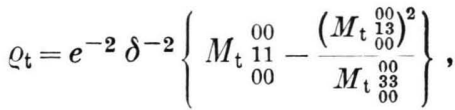

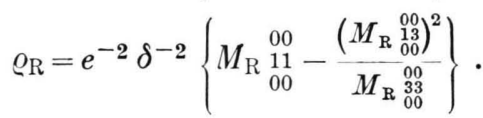

Es tritt demnach folgende Abweichung von der Additivität des elektrischen Widerstandes auf

$$
\begin{aligned}
& (\Delta \varrho)_{\mathrm{Matt}}=
\end{aligned}
$$

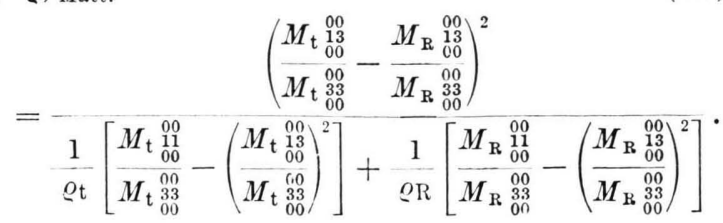

Wie aus der Tabelle für die Matrixelemente $M_{\mathrm{t}} \begin{gathered}m o \\ n l \\ 00\end{gathered}$ $\mathrm{zu}$ entnehmen ist, ist der in der eckigen Klammer hinter $1 / \varrho_{t}$ stehende Ausdruck nahezu gleich eins. 
Da über die Art der Gitterfehlstellen, die den Restwiderstand verursachen, meist sehr wenig bekannt ist, machen wir dieselbe Näherung auch in der zweiten Klammer. Es ist dann

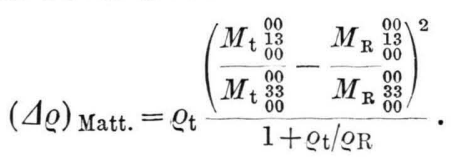

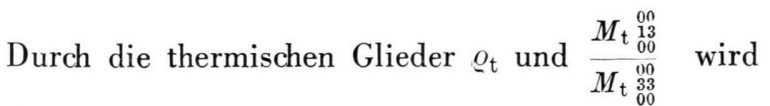
die Abweichung von der Matthiessenschen Regel temperaturabhängig, und zwar können wir zwei Fälle unterscheiden:

1. Wenn der thermische Widerstand klein gegenüber dem Restwiderstand ist, nimmt $(\Delta \varrho)_{\text {Matt. }}$ linear mit $\varrho_{\mathrm{t}} \mathrm{zu}$.

2. Wenn der thermische Widerstand groß gegenüber dem Restwiderstand ist, ergibt sich die konstante Abweichung

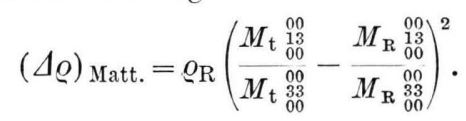

Wie groß eventuell diese Abweichungen werden können, soll an einem Beispiel erläutert werden. MAGNUson, Palmer und Koenler ${ }^{45}$ haben $99,999 \%$-Kupfer, das einen Restwiderstand von $1,30 \cdot 10^{-9} \Omega$ cm be$\mathrm{sa} \beta$, mit Deuteronen bestrahlt und dadurch eine Widerstandszunahme von $5,61 \cdot 10^{-8} \Omega \mathrm{cm}$ erreicht. Es wurde dann die Erholung der Strahlungsschädigung bei bestimmten Temperaturen untersucht. In einem Restwiderstands-Zeitdiagramm ist die Erholung durch eine abfallende Kurve sichtbar. Bei Wechsel zu einer höheren Erholungstemperatur treten außerdem noch kleine positive Sprünge auf. Die Autoren haben diesen Effekt, der nicht mit der Erholung zusammenhängt, weil er nur von der Temperatur und nicht von der Zeit abhängig ist, einer Änderung der Debye-Temperatur durch die Bestrahlung zugeschrieben. Es läßt sich nun zeigen, daß wir ihn zwanglos durch Abweichungen von der Matthiessenschen Regel deuten können.

Bei tiefen Temperaturen ist das Anisotropieverhältnis $M_{\mathrm{t}}{ }_{00}^{00} / M_{\mathrm{t}_{00}^{33}}^{00}$ so groß, daß wir das zweite von den statischen Gitterfehlern herrührende Glied vernachlässigen können. Für verschiedene Temperaturen haben wir die Größe der Abweichungen von der

45 G. D. Magnuson, W. Palmer u. J. S. Koehler, Phys. Rev. 109, 1990 [1958].
Matthiessenschen Regel für die bestrahlte und unbestrahlte Probe berechnet. Die Temperaturabhängigkeit des spezifischen Widerstandes $\varrho_{t}$ haben wir den Messungen von Berman und MacDonald ${ }^{46}$ entnommen. Für das Anisotropieverhältnis $M_{\mathrm{t}}{ }_{00}^{00} / M_{\mathrm{t}}{ }_{00}^{00}$ haben wir den Wert für $20,4{ }^{\circ} \mathrm{K}$ eingesetzt. Diese Annahme ist zunächst nicht voll gerechtfertigt, weil der obige Quotient ziemlich temperaturabhängig ist. Wie wir noch sehen werden, müssen wir jedoch bei höheren Temperaturen die Abweichungen der FermiOberfläche von der Kugelform berücksichtigen, so daß der Fehler, den wir durch obige Vereinfachung begehen, nicht sehr groß sein wird. Wie man aus Abb. 7 sieht, kann mit Hilfe der Anisotropie des

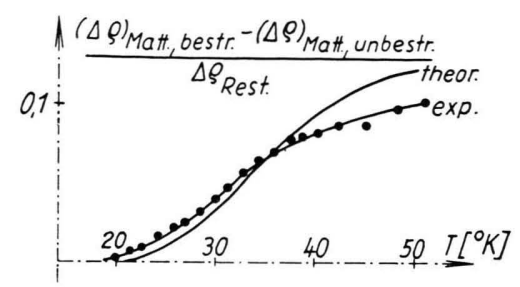

Abb. 7. Differenz der Abweichungen von der Matthiessenschen Regel bei einer bestrahlten und unbestrahlten Probe in Abhängigkeit von der Temperatur.

Streumechanismus sowohl der Betrag als auch die Temperaturabhängigkeit der Sprünge qualitativ erklärt werden.

\section{c) Änderung des elektrischen Widerstandes im Magnetfeld}

In gleicher Weise kann man die Änderung des elektrischen Widerstandes im Magnetfeld bei tiefen Temperaturen quantitativ verstehen. Zunächst fällt in Tab. 2 auf, daß bei $20,4^{\circ} \mathrm{K}$ schon mit einem magnetischen Querfeld von $800 \mathrm{Gau}$ eine Erhöhung des elektrischen Widerstandes um den Faktor 2 erreicht werden kann. Da bei der Ableitung des Variations-Iterationsverfahrens vorausgesetzt wurde, daß das Magnetfeld nur eine Störung der thermischen Streuung der Elektronen ist, können wir mit den abgeleiteten Beziehungen, die für die Änderung des elektrischen Widerstandes eine quadratische Abhängigkeit von der Feldstärke voraussagen, nur den Bereich $0<H<300 \mathrm{Gau}$ beschreiben. Um

46 R. Berman u. D. K. C. MacDonald, Proc. Roy. Soc., Lond. A 211, 122 [1952]. 
noch höhere Feldstärken erfassen zu können, müßten wir außer den quadratischen Gliedern auch noch höhere Potenzen des Magnetfeldes berücksichtigen. Dieses Ergebnis steht in Übereinstimmung mit den Messungen von Grüneisen und Adenstedt ${ }^{47}$, die die magnetische Widerstandsänderung eines natürlich gewachsenen Einkristalls mit dem Widerstandsverhältnis $\varrho_{T}^{(0)} / \varrho_{T_{0}}^{(0)}=8,4 \cdot 10^{-4}$ bei $20,4{ }^{\circ} \mathrm{K}$ bestimmt haben. Sie fanden nämlich heraus, daß sich die Änderung des elektrischen Widerstandes schon von kleinen Feldstärken ab durch eine lineare Abhängigkeit beschreiben läßt. Mit einem magnetischen Querfeld von $H=300 \mathrm{Gauß}$ haben die Autoren eine Erhöhung des Widerstandes um $\Delta Q=1,20 \cdot 10^{-10} \Omega \mathrm{cm}$ unabhängig von der Orientierung des Magnetfeldes gegenüber den Kristallachsen erzielt. Der theoretische Wert für diese Feldstärke ist winkelabhängig und schwankt zwischen 0,8 und $0,95 \cdot 10^{-10} \Omega \mathrm{cm}$. Für diese Feldstärke ist also befriedigende Übereinstimmung zwischen Theorie und Experiment vorhanden. Die Berechnung der Widerstandsänderung für höhere Feldstärken versagt aus den oben angeführten Gründen. Eine Prüfung der theoretischen Ergebnisse - insbesondere die Verkleinerung der HalL-Konstanten um den Faktor 2 bzw. 4 gegenüber dem Wert des freien Elektronengases - an weiterem experimentellem Material konnte nicht durchgeführt werden, weil bei den meisten Arbeiten ${ }^{48,49}$ der Restwiderstand weitgehend den thermischen Widerstand überwogen hat. Da der Restwiderstand vor allem durch mehr isotrop streuende Gitterfehler als es die Gitterschwingungen sind, verursacht wird, wird dadurch der Betrag der Widerstandsänderung im Magnetfeld weitgehend herabgesetzt.

Bei der Temperatur von $77,4{ }^{\circ} \mathrm{K}$ und Zimmertemperatur liegen nur Messungen an polykristallinem Material vor. Für $77,4{ }^{\circ} \mathrm{K}$ kann man aus den Messungen von Kapitza ${ }^{50}$ folgende Werte entneh- men: $A_{\perp}=10^{-11} \mathrm{Oe}^{-2}$ und $A_{\|}=5 \cdot 10^{-12} \mathrm{Oe}^{-2}$. Für die Änderung des Widerstandes im transversalen Magnetfeld gibt Grunmach ${ }^{51}$ bei Zimmertemperatur den Wert $A_{\perp}=4 \cdot 10^{-13} \mathrm{Oe}^{-2}$ an.

Die theoretischen Werte bei $T=77,4{ }^{\circ} \mathrm{K}$ und $T=273,15^{\circ} \mathrm{K}$ sind also um den Faktor $30 \mathrm{bzw}$. 300 zu klein. Diese Diskrepanz kommt dadurch zustande, daß bei hohen Temperaturen die wesentliche Anisotropie der Übergangswahrscheinlichkeit nicht von den thermischen Gitterschwingungen, sondern von der Abweichung der Elektronenenergiefläche von der Kugelgestalt herkommt, während bei tiefen Temperaturen durch nicht angeregte Gitterschwingungen eine Anisotropie verursacht wird, die den Einfluß der nichtsphärischen Energieflächen überdeckt. Die Berechnung der magnetischen Widerstandsänderung bei nichtsphärischen Energieflächen ist äußerst kompliziert und wird zur Zeit durchgeführt.

Herrn Professor Dr. U. Dehlinger danke ich sehr für sein förderndes Interesse an der vorliegenden $\mathrm{Ar}$ bei. Mein besonderer Dank gilt Herrn Prof. Dr. A. SeEGER für die Anregung zu diesen Untersuchungen und für viele eingehende Diskussionen. Der $\mathrm{D}$ e u $\mathrm{t} \mathrm{s} \mathrm{ch}$ en Forschungsgemeinschaft habe ich für finanzielle Unterstützung zu danken.

\section{Anhang}

Umformung des Ausdrucks $B_{\sigma \sigma}^{(0)}\left(\mathfrak{r}, \mathfrak{r}^{\prime}\right) \cdot K_{2}\left(\mathfrak{r}-r^{\prime}\right)$ auf bikubisch-sphärische Harmonische.

Der in Abschnitt 3 auftretende Ausdruck

$$
B_{\sigma \sigma}^{(0)}\left(\mathfrak{r}, \mathrm{r}^{\prime}\right) \cdot K_{2 \varrho}\left(\mathrm{r}-\mathrm{r}^{\prime}\right)
$$

ist eine Funktion der beiden Variablen $r$ und $r^{\prime}$, besitzt kubische Symmetrie und gehört zur Darstellung $\mathfrak{D}^{(2 \varrho)}$. Wie wir weiter unten zeigen werden, erfüllt er auch die von Hobsos ${ }^{52}$ angeführten Bedingungen, die für eine Entwicklung nach Kugelflächenfunktionen vorausgesetzt werden müssen. Wir können ihn daher in folgender Weise

$$
B_{\sigma \sigma}^{(0)}\left(\mathrm{r}, \mathrm{r}^{\prime}\right) \cdot K_{2 \varrho}\left(\mathrm{r}-\mathrm{r}^{\prime}\right)=\sum_{n, l} k_{n l}^{(2 \varrho ; \sigma,+)}\left(r, r^{\prime}\right) \cdot B_{n l}^{(2 \varrho,+)}\left(\vartheta, \varphi ; \vartheta^{\prime}, \varphi^{\prime}\right)+\sum_{n, l} k_{n l}^{(2 \varrho ; \sigma,-)}\left(r, r^{\prime}\right) \cdot B_{n l}^{(2 \varrho,-)}\left(\vartheta, \varphi ; \vartheta^{\prime}, \varphi^{\prime}\right)
$$

nach bikubisch-sphärischen Harmonischen entwickeln. Im allgemeinen Fall $r \neq r^{\prime}$ müssen dabei die Entwicklungskoeffizienten $k_{n l}^{(20 ; \sigma,+)}\left(r, r^{\prime}\right)$ symmetrisch und die

47 E. Grüneisen u. H. Adenstedt, Ann. Phys., Lpz. 31, 714 [1938].

48 P. Jongenburger, Conf. de Physique des Basses Températures, Paris, No. 47, 411 [1955].

49 H. G. van Bueren, Thesis Leyden [1956].
Entwicklungskoeffizienten $\quad k_{n}^{(2 \varrho ; \sigma,-)}\left(r, r^{\prime}\right)$ antisymmetrisch in den beiden Variablen $r$ und $r^{\prime}$ sein, weil $K_{2}\left(r-r^{\prime}\right)$ die beiden Vektoren $r$ und $r^{\prime}$ in symmetri-

${ }^{50}$ P. Kaptrza, Proc. Roy. Soc., Lond. A 123, 292 [1929].

${ }^{51}$ L. Grunmach, Ann. Phys., Lpz. 22, 141 [1907].

52 E. W. Hosson, The Theory of Spherical and Ellipsoidal Harmonics, University Press, Cambridge 1931. 
scher Weise enthält. Im Zusammenhang mit der in Abschnitt 3 benötigten Entwicklung können wir uns auf den Spezialfall $r=r^{\prime}$ beschränken, bei dem alle $k_{n l}^{(2 \varrho ; \sigma,-)}\left(r, r^{\prime}\right)$ verschwinden und die symmetrischen Koeffizienten außerdem noch unabhängig von $r$ werden.
Für die weitere Ableitung benützen wir die Eigenschaft der kubischen Harmonischen, daß sie sich durch homogene Polynome vom Grad $2 \varrho$ darstellen lassen, welche invariant gegenüber allen Symmetrieoperationen der kubischen Gruppe sind.

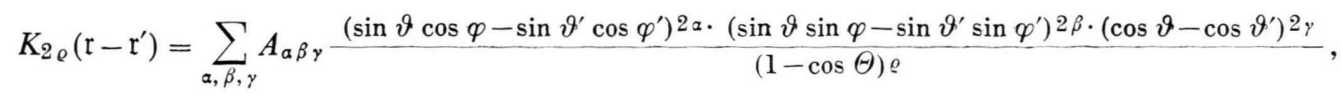

wobei

$$
\alpha+\beta+\gamma=\varrho \quad \text { und } \quad|\mathrm{r}|=\left|\mathrm{r}^{\prime}\right|
$$

angenommen wurde.

Der im Zähler stehende Ausdruck besitzt schon kubische Symmetrie und läßt sich daher nach bikubischsphärischen Harmonischen entwickeln, und zwar muß - wie man sich leicht überzeugen kann - folgende Entwicklung möglich sein

$$
K_{2 \varrho}\left(\mathfrak{r}-\mathbf{r}^{\prime}\right)=\sum_{\tau=0}^{\varrho} \frac{a_{\tau, 2 \varrho-\tau}^{(2 \varrho ;+)} \cdot B_{\tau, 2 \varrho-\tau}^{(2 \varrho ;+)}}{(1-\cos \Theta) \varrho}=\sum_{n, l} k_{n l}^{(\varrho ; 0,+)} \cdot B_{n i}^{(\varrho,+)}\left(\vartheta, \varphi ; \vartheta^{\prime}, \varphi^{\prime}\right)
$$

und allgemein

$$
B_{\sigma \sigma}^{(0)} K_{2 \varrho}\left(\mathrm{r}-\mathrm{r}^{\prime}\right)=B_{\sigma \sigma}^{(0)} \sum_{\tau=0}^{\varrho} \frac{a_{\tau, 2 \varrho-\tau}^{(2 \varrho ;+)} \cdot B_{\tau, 2 \varrho-\tau}^{(2 \varrho ;+)}}{(1-\cos \Theta) \varrho}=\sum_{n, l} k_{n l}^{(2 \varrho, \sigma,+)} \cdot B_{n l}^{(2 \varrho,+)}\left(\vartheta, \varphi ; \vartheta^{\prime}, \varphi^{\prime}\right) .
$$

Wegen der Integraleigenschaften der bikubisch-sphärischen Harmonischen sind die Entwicklungskoeffizienten $k_{n i}^{(o, \sigma,+)}$ bestimmt durch

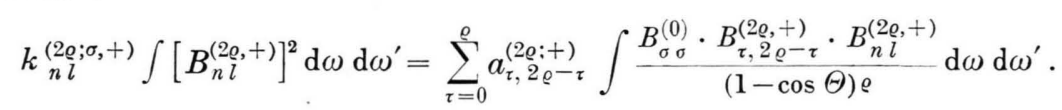

Die Auswertung des auf der rechten Seite stehenden Integrals scheint wegen des Faktors $(1-\cos \Theta) \varrho$ im Nenner äußerst schwierig zu sein; ja es ist sogar fraglich, ob das Integral überhaupt einen endlichen Wert besitzt. Mit Hilfe eines Grenzübergangs $\vartheta \rightarrow \vartheta^{\prime}$ und $\varphi \rightarrow \varphi^{\prime}$; d. h. $\cos \Theta \rightarrow 1$, können wir zeigen, daß der Integrand beschränkt bleibt, jedoch an der Stelle $\cos \Theta=1$ unendlich vieldeutig wird. Nach .Hobson wird daher die oben angegebene Entwicklung, wenn $\cos \Theta \neq 1$ ist, gleichmäßig gegen den jeweiligen Funktionswert konvergieren. An der Stelle $\cos \Theta=1$ wird dagegen die Entwicklung einen Wert annehmen, der ein Mittelwert aus den unendlich vielen Funktionswerten ist. Für die weitere Berechnung des Integrals entwickeln wir das Produkt $B_{\tau, 2 \varrho-\tau}^{(2 \varrho,+)} \cdot B_{n l}^{(2 \varrho,+)}$ nach bikubischen, sphäriscken Harmonischen. Nach dem Vektoradditionsmodell treten hierbei Funktionen auf, die zur Darstellung $\boldsymbol{D}^{(2 L)}$ gehören, wobei $0 \leqq L \leqq 2 \varrho$ ist

$$
B_{\tau, 2 \varrho-\tau}^{(2 \varrho,+)} \cdot B_{n l}^{(2 \varrho,+)}=\sum_{L=0}^{2 \varrho} \sum_{v, \lambda} c_{v \lambda}^{(L)} \cdot B_{v i}^{(L,+)}
$$

Setzen wir nun die Entwicklung des Produkts $B_{\tau, 2 \varrho-\tau}^{(2 \varrho,+)} \cdot B_{n l}^{(2 \varrho,+)}$ in das obige Integral ein, so ergibt sich wegen der Orthogonalitätsrelation, daß nur die Glieder mit $L=0$ einen Beitrag liefern.

$$
\begin{aligned}
k_{n l}^{(2 \varrho ; \sigma,+)} \int\left[B_{n l}^{(2 \varrho,+)}\right]^{2} \mathrm{~d} \omega \mathrm{d} \omega^{\prime} & =\sum_{\tau=0}^{\varrho} a_{\tau}^{(2 \varrho,+)}, \sum_{\nu} c_{\nu \nu}^{(0)} \int \frac{B_{\sigma \sigma}^{(0)} \cdot B_{\nu \nu}^{(0)}}{(1-\cos \Theta) \varrho} \mathrm{d} \omega \mathrm{d} \omega^{\prime} \\
& =8 \pi^{2} \sum_{\tau=0}^{\varrho} a_{\tau, 2 \varrho}^{(2 \varrho,+)} \sum_{\nu} c_{\nu \nu}^{(0)} \int_{-1}^{1} \frac{P_{\nu}(x) \cdot P_{\sigma}(x)}{(1-x) \varrho} \mathrm{d} x .
\end{aligned}
$$

Der Koeffizient $C_{\nu,}^{(0)}$ besitzt den Wert

$$
C_{\nu \nu}^{(0)}=\frac{2 \nu+1}{2} \frac{1}{8 \pi^{2}} \int B_{\tau, 2 \varrho-\tau}^{(2 \varrho,+)} \cdot B_{n l}^{(2 \varrho,+)} \cdot B_{\nu \nu}^{(0)} \mathrm{d} \omega \mathrm{d} \omega^{\prime}=\frac{2 \nu+1}{16 \pi^{2}} b_{n, l}^{(v, 2 \varrho)}, \underset{n, \tau}{, 2 \varrho} \int\left[B_{\tau, 2 \varrho-\tau}^{(2 \varrho,+)}\right]^{2} \mathrm{~d} \omega \mathrm{d} \omega^{\prime} .
$$

Wegen der Eigenschaft der Koeffizienten $b_{\tau}^{(v, 2 \varrho)}, \underset{n}{2} \varrho^{-\tau}$ ist $C_{\nu \nu}^{(0)}$ nur dann von Null verschieden, wenn die Ungleichung $\frac{1}{2}(n+l)-\varrho \leqq \nu \leqq \frac{1}{2}(n+l)+\varrho$ erfüllt ist. 
Damit wird

$k_{n, l}^{(2 \varrho, \sigma,+)} \int\left[B_{n l}^{(2 \varrho,+)}\right]^{2} \mathrm{~d} \omega \mathrm{d} \omega^{\prime}=\sum_{\tau=0}^{\rho} a_{\tau, 2 \varrho-\tau}^{(2 \varrho,+)} \int\left[B_{\tau, 2 \varrho \varrho^{-\tau}}^{(2 \varrho,+)}\right]^{2} \mathrm{~d} \omega \mathrm{d} \omega^{\prime}$

$$
\underset{\nu=[(n+l) / 2]-\varrho}{[(n+l) / 2]+\varrho} \frac{2 v+1}{2} b_{\substack{n, 2 \\ n, l}}^{(v, 2 \varrho)} \int_{-1}^{1} \frac{P_{\sigma}(x) \cdot P_{\nu}(x)}{(1-x) \varrho} \mathrm{d} x
$$

Wie wir schon gezeigt haben, muß das obige Integral einen endlichen Wert haben. Dies ist nur möglich, wenn folgende Umordnung durchführbar ist

$$
\sum_{\tau=0}^{\varrho} a_{\tau, 2 \varrho-\tau}^{(2 \varrho,+)} \int\left[B_{\tau, 2 \varrho-\tau}^{(2 \varrho,+)}\right]^{2} \mathrm{~d} \omega \mathrm{d} \omega^{\prime} \sum_{v=[(n+l) / 2]-\varrho}^{[(n+l) / 2]+\varrho} \frac{2 v+1}{2} b b_{n l}^{(v, 2 \varrho)} \varrho^{(, 2)} P_{v \cdot}(x)=(1-x) \stackrel{(n+l) / 2}{\sum_{x=0}^{\prime}} d_{n l}^{\varkappa} P_{\varkappa}(x) .
$$

Man erhält somit endgültig

$$
k_{n l}^{(2 \varrho ; \sigma,+)}=\left\{\begin{array}{cr}
\frac{2}{2 \sigma+1} \int\left[B_{n l}^{(2 \varrho,+)}\right]^{2} \mathrm{~d} \omega \mathrm{d} \omega^{\prime} & 0 \leq \sigma \leq \frac{n+l}{2}, \\
0 & \sigma>\frac{n+l}{2} .
\end{array}\right.
$$

\section{NOTIZEN}

\section{Über ein Spinormodell in der Quantentheorie nichtlinearer Wellengleichungen}

\section{Von K. LaDÁnYi}

Forschungsgruppe für Theoretische Physik der Ungarischen Akademie der Wissenschaften, Budapest

(Z. Naturforschg. 14 a, 580-581 [1959]; eingegangen am 12. Februar 1959)

Wie bekannt, ist in der zum Studium der wichtigsten Züge der Heisenbergschen nichtlinearen Feldtheorie ${ }^{1}$ eingeführten Lagrange-Funktion

$$
L=\psi^{+} \gamma_{\mu} \partial_{\mu} \psi+\frac{l^{2}}{2}\left(\psi^{+} \psi\right)\left(\psi^{+} \psi\right)
$$

der Isotopenspin nicht berücksichtigt. In einer späteren Arbeit zeigten Heisenberg und $\mathrm{Pauli}^{2}{ }^{2}$, daß die LAGRANGE-Funktion

$$
L^{\prime}=\psi^{+} \gamma_{\mu} \partial_{\mu} \psi \pm \frac{l^{2}}{2}\left(\psi^{+} \gamma_{\mu} \gamma_{5} \psi\right)\left(\psi^{+} \gamma_{\mu} \gamma_{5} \psi\right)
$$

den Grund einer künftigen Theorie der Elementarteilchen bilden kann. Man kann zeigen, daß die Erhaltung der Baryonenzahl und der Ladung gesichert, und daß $L^{\prime}$ invariant gegen die mit der Isotopenspingruppe isomorphen PaULI-Transformation ist ${ }^{3,4}$. Von einigen einfachen Annahmen ausgehend wird im folgenden das qualitative Modell der Theorie der Elementarteilchen auf Grund einer anderen Lagrange-Funktion konstru-

1 W. Heisenberg, Rev. Mod. Phys. 29, 269 [1957]. In dieser Arbeit sind auch weitere Literaturangaben zu finden.

2 W. Heisenberg u. W. Pauli, On the Isospingroup in the Theory of the Elementary Particles, 1958. Prepint.

3 W. Pauli, Nuovo Cim. 6, 204 [1957].

4 G. Gürsey, Nuovo Cim. 7, 411 [1958]. iert. Diese Annahmen können folgendermaßen zusammengefaßt werden:

1. Das nichtlineare Glied der Lagrange-Funktion entspricht einer universalen Fermi-Wechselwirkung.

2. Die Lagrange-Funktion zeigt keine Invarianzeigenschaften, die in der Natur nicht vorkommen.

3. In der Theorie treten nur zwei Spinoren mit vier Komponenten $\psi_{1}$ und $\psi_{2}$ auf.

4. Die Lagrange-Funktion ist gegen die Transformationen

$$
\psi_{2}^{\prime}=e^{i \beta} \psi_{2} \quad \psi_{1}^{\prime}=e^{i \alpha} \psi_{1},
$$

invariant.

Im Einklang mit unseren Grundannahmen wird jetzt die Lagrange-Funktion

$L=\psi_{i}^{+} \gamma_{\mu} \partial_{\mu} \psi_{i}+\lambda_{i j k l}\left(\psi_{i}^{+} \gamma_{\mu} a \psi_{j}\right)\left(\psi_{k}^{+} \gamma_{\mu} \bar{a} \psi_{l}\right)$

untersucht, wo nur die Koeffizienten $\lambda_{1122}, \lambda_{2211}, \lambda_{1111}$, $\lambda_{1221}, \lambda_{2112}$ und $\lambda_{2222}$ von Null verschieden sind, und weiterhin

$$
a=A+B \gamma_{5}, \quad \bar{a}=\bar{A}+\bar{B} \gamma_{5}
$$

ist.

Die nähere Untersuchung der Annahmen

$$
A=B=\bar{A}=-\bar{B}=1,
$$

scheint zweckmäßig zu sein. Es ist leicht zu zeigen, $\mathrm{da} a$ die Invarianz

$$
\left(\begin{array}{l}
\psi_{1}^{\prime} \\
\psi_{2}^{\prime}
\end{array}\right)=e^{i \alpha \gamma_{5}}\left(\begin{array}{l}
\psi_{1} \\
\psi_{2}
\end{array}\right)
$$

gesichert ist. Wir heben aber hervor, daß im Falle der Kopplung (6) die Lagrange-Funktion gegen die Transformationen

$$
\begin{aligned}
& \psi_{1}{ }^{\prime}=\gamma_{5} \dot{\psi}_{1}, \\
& \psi_{2}{ }^{\prime}=\gamma_{5} \psi_{2}
\end{aligned}
$$

